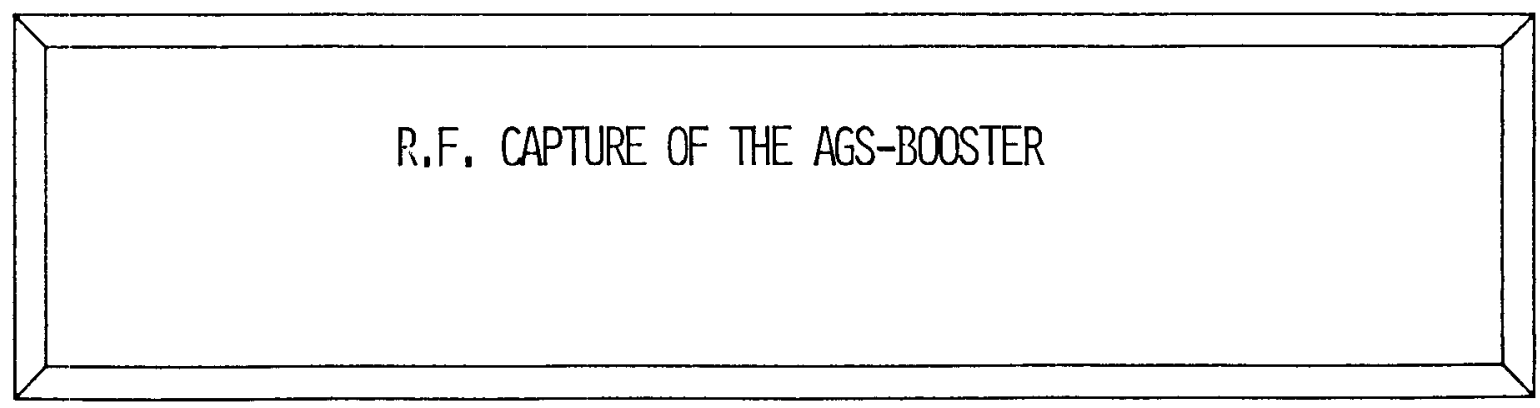

$A D$

Booster Technical Note

No. 115

J. WEI, S,Y, LEE AND A,G, RUGGIERO

APRIL 8, 1988

ACCELERATOR DEVELOPMENT DEPARTMENT

Brookhaven National Laboratory

Upton, N.Y. 11973 


\title{
R.F. CAPTURE OF THE AGS-BOOSTER
}

\author{
J. Wei, S. Y. Lee and A. G. Ruggiero \\ Accelerator Development Department \\ Brookhaven National Laboratory \\ Upton, New York 11973
}

\begin{abstract}
Computer simulation of the longitudinal phase space dynarnics showes that the space charge induced fields contribute considerable effects to the capture and the distribution of the particles. Statistical analysis is done to ensure that the calculation of the space charge fields is reliable.

With the $10 \mathrm{~ms}$ programming of the R.F. voltage $\hat{V}$ and the synchronous phase $\phi_{s}$, $94 \%$ of the 20 turns of the multi-turn injected $\mathrm{Au}^{+33}$ heavy-ion beam can be captured in a longitudinal phase space area of $0.06 \mathrm{ev} \cdot \mathrm{sec} / \mathrm{amu}$. If a similar R.F. programming is performed for the proton injection, $96 \%$ of the proton beam can be effectively capturcd in the Booster with a longitudinal phase space area of $1.0 \mathrm{ev} \cdot \mathrm{sec}$.
\end{abstract}




\section{INTRODUCTION}

During the injection period, the Coulombic space charge field induced by the circulating beam contribute considerable effects to the beam capture process on the Booster ${ }^{2}$. The computer program ${ }^{3}$ which was originally developed to simulate the transverse and the longitudinal phase space dynamics during the multi-turn injection and the capture is thus modified to take into account the longitudinal space charge effect.

The simulation for the space charge force is accomplished by dividing the bunch into bins of finite length in the longitudinal space. To obtain a reliable space charge force, the bin size should not be too large. The number of particles per bin should not be too small. Besides the mean field space charge force, the program should be able to study the collective phenomena. In order to include the collective instability, the bin length used for the calculation of the space charge induced voltage should be comparable to the collective wave length. With the bin length specified, a certain number of representative particles is required for the calculation to reach an accuracy level. The reliability of the simulation result essencially depends on the statistical accuracy of the calculation of the space charge voltage .

In this paper, we shall report on the study of the multi-turn injection for the AGSbooster. The method and the statistical analysis on the calculation of the space charge effect is discussed in Section II. The results of the simulation of the multi-turn injection and the R.F. capture of the beam of heavy ions and protons are given in Section III. Discussions and conclusions are given in Section IV. 


\section{METHOD ON THE SPACE CHARGE CALCULATION}

\section{A. Equations of Motion}

Once the beam of the particles is injected into the machine, the motion of each individual particle in the longitudinal phase space $\left(\phi, \frac{\Delta E}{\Omega_{0}}\right)$ is described by the difference equations

$$
\begin{gathered}
\delta_{n+1}=\delta_{n}+\frac{Z e \hat{V}_{n}}{A m_{0} c^{2} \gamma_{s, n}} \cdot\left(\sin \phi_{n}-\sin \phi_{s, n}\right)+\Delta_{s . c .}\left(\phi_{n}\right)_{n}+\Delta_{Z}\left(\phi_{n}\right)_{n} \\
\phi_{n+1}=\phi_{n}+\frac{2 \pi h \eta_{s}}{\beta_{s, n}^{2}} \cdot \delta_{n+1}
\end{gathered}
$$

where

$\eta_{s}=\frac{1}{\gamma_{t}^{2}}-\frac{1}{\gamma_{s}^{2}}$

$\gamma_{s}=\left(1-\beta_{s}^{2}\right)^{-\frac{1}{2}}$ synchronous energy of the particle in $m_{0} c^{2}$ units

$\delta=\frac{\Delta E}{E_{s}}=\frac{\Delta \gamma}{\gamma_{s}}=\beta_{s}^{2} \frac{\Delta}{p_{s}}$, relative deviation of the particle energy

$\phi=$ deviation of the particle R.F. phase from $\phi_{s}$

$h=$ R.F. harmonic number

$Z=$ charge carried by the particle

$A=$ atomic number of the particle

$\Delta_{s . c .}=$ relative energy gain per turn due to the space charge fields

$\Delta_{z}=$ relative energy gain per turn due to other kinds of impedance

$n($ subscript $)=$ revolution number.

If the linear density $\lambda(\phi)$ of the beam changes slowly ${ }^{1,4}$ within distance comparable to the diameter of the vacuum chamber $2 b$,

$$
\frac{2 b}{\lambda} \frac{\partial \lambda}{\partial s} \ll 1
$$

the $\Delta_{\text {s.c. }}\left(\phi_{n}\right)$ can be represented by the mean field expression

$$
\Delta_{\text {s.c. }}\left(\phi_{n}\right)=\frac{e V_{s . c .}\left(\phi_{n}\right)}{A m_{0} c^{2} \gamma_{s}}=\frac{Z_{0} g_{0} R_{0} c Z e}{2 A m_{0} c^{2} \gamma_{s}^{3}} \cdot \frac{\partial \lambda\left(\phi_{n}\right)}{\partial s}
$$


where $Z_{0}=\left(\varepsilon_{0} c\right)^{-1}=377 .(o h m), R_{0}$ is the average radius of curvature. $g_{0}$ is the geometric factor, $g_{0}=1+2 \ln \frac{b}{a}$ for a cylindrical geometry.

The $\Delta_{R}\left(\phi_{n}\right)$ of the resistive wall coupling impedance can be similarly represented by the mean field expression

$$
\Delta_{R}\left(\phi_{n}\right)=\frac{e V_{R}\left(\phi_{n}\right)}{A m_{0} c^{2} \gamma_{s}}=\frac{R_{w} \beta_{0} R_{0} c Z e}{A m_{0} c^{2} \gamma_{s}} \cdot \lambda\left(\phi_{n}\right)
$$

where $R_{w}$ is the wall coupling resistance.

The voltage induced by the space charge $V_{\text {s.c. }}(\phi)$ is found by evaluating the derivative of the density distribution function of the beam in the $\phi$ space ${ }^{5}$

$$
V_{\text {s.c. }}(\phi)=\frac{Z^{2} h^{2} g_{0} Z_{0} c e}{2 R_{0} \gamma_{s}^{2}} \cdot \frac{\partial\left(N_{0} f(\phi)\right)}{\partial \phi}
$$

where $N_{0}$ is the total number of particles per bunch, $N_{0} f(\phi)$ is the number of particles per unit $\phi$ around $\phi_{s}$. Let $N_{b}$ denote the number of bins of the $2 \pi$ phase period,

$$
N_{b}-\frac{2 \pi R_{0}}{h l_{b}}
$$

where $l_{b}$ is the bin length. To study the effect of microwave instability the bin length should be of the order of the wave length up to the microwave cutoff frequency ${ }^{6,7}$,i.e., $l_{b} \sim b$.

\section{B. Estimation of the Statistical Deviation}

If $N_{t}$ is the total number of the representative particles used in the simulation, $\Delta \phi$ is the bin size in $\phi$ space, the density function $f(\phi)$ at the ith bin is

$$
f(\phi)_{i}=\begin{gathered}
1 \\
N_{t}
\end{gathered}\left(\begin{array}{c}
\left(\frac{N_{t}}{N_{h}} g_{i}\right) \\
\Delta \phi
\end{array}\right)
$$

where $g_{i}$ is the weighting factor,

$$
\sum_{i=1}^{N_{b}} \frac{g_{i}}{N_{b}}=1, \sum_{i=1}^{N_{b}} f(\phi)_{i} \Delta \phi=1
$$


and

$$
\frac{\partial f(\phi)}{\partial \phi}=\frac{1}{N_{t}}\left(\frac{\left(\frac{N_{t}}{N_{h}} g_{i}\right)}{\Delta \phi}-\frac{\left(\frac{N_{t}}{N_{b}} g_{i-1}\right)}{\Delta \phi}\right) \cdot \frac{1}{\Delta \phi} .
$$

The statistical fluctuation on the number of the representative particles at the ith bin is

$$
\Delta\left(\frac{N_{t}}{N_{b}} g_{i}\right)=\sqrt{\frac{N_{t}}{N_{b}} g_{i}} .
$$

'Thus the statistical deviation of $V_{\text {s.c. }}$ becomes

$$
\Delta\left(V_{s . c .}\right) \sim \sqrt{g_{i}} \cdot \sqrt{N_{b}^{3}}
$$

The statistical analysis indicates that the deviation of the space charge voltage is inversely proportional to the square root of the product of the total number of the representative particles and the bin length to the cubic power. Therefore, if the bin length is decreased to take into account the effect from the higher frequency, the total number of particles used in the simultion must be increased to the cubic power of $N_{b}$ in order to retain the same statistical accuracy. The behaviour of equation (12) is observed by systematically evaluating the averaged statistical deviation as a function of $\left(N_{b}, N_{t}\right)$, as being discussed in Appendix I ( Figure $1 \sim$ Figure 5 ).

If instead of the space sharge effect, other impedance induced effects are dominating, the statistical behaviour would be different. The voltage induced by resistive wall impedance is discussed in Appendix II. In this case we notice that as long as the number of the representative particles per bin remains constant, the same statistical accuracy is always achieved.

\section{The Computer Program}

The computer program is developed to simulate the multi-turn injection and the R.F. capture process. Initially the program randomly generates a d.c. beam with the specified injection energy and the energy spread. Relalive to the injecting beam, the R.F. bucket gradually moves up due to the acceleration. The movement of the R.F. bucket, the synchrotron oscillation motion and the increasing space charge effect make 
the particle distribution in the $\phi$ space no longer uniform by the time the multi-turn injection is completed. The particles are tracked further on a turn-by-turn basis to higher energy. The R.F. voltage and the synchronous phase can be programmed during the entire process. The number of the particles remaining inside the R.F. bucket is calculated any time upon request. The distribution in the longitudinal phasc spacc, the projection on the phase and the momentum axis and the voltage distribution of the spare. charge are also printed out. The beam populated phase space area and the statistical error of the calculation can thus be found.

The space charge and the resistive coupling impedance can both be included in the simulation. The treatment of the space charge followes Section II.a and II.b, except that the three-point formula

$$
f_{p}^{\prime}=f^{\prime}\left(\phi_{0}+p h\right)=\frac{1}{h}\left(\left(p-\frac{1}{2}\right) f_{-1}-2 p f_{0}+\left(p+\frac{1}{2}\right) f_{1}\right)+R_{2}^{\prime}
$$

is used to decide the quantity $\underset{\partial \phi}{\partial f(\phi)}$. Calculating $\underset{\partial \phi}{\partial f(\phi)}$ by using the information in every t, wo nearby bins, i.e. by the two-point formula, may easily give fluctuation and artificial wiggling in the phase space diagram. The three-point formula of eq.(13) greatly improves the result.

\section{RESULTS OF THE R.F. CAPTURE ON THE BOOSTER}

\section{A. R.F. Capture of the Heavy Ions}

The beam of the heavy ions comes from the Tandem Van De Graaff accelerator with kinetic energy spread of $10^{-3}$. Each single pulse of the $300 \mu$ s heavy ion ${ }^{197} \mathrm{Au}^{+33}$ is able to provide more than 20 turns of "ribbon" beam. For the ${ }^{197} \mathrm{Au}{ }^{+33}$ beam, an intensity of $2.2 \cdot 10^{9}$ per bunch is requested upon the extraction on the Booster.

During the injection and the capturing process, the R.F. voltage and the synchronous phase are adiabatically raised in $10 \mathrm{~ms}$ from $1.6 \mathrm{kV}$ and $0.001 \mathrm{rad}$. to $17 \mathrm{kV}$ and $0.6 \mathrm{rad}$. respectively. Assuming $h=3$, the evolution in the longitudinal phase space without the 
space charge is shown in Figure 6. More than $94 \%$ of the $\mathrm{Au} \mathbf{u}^{+33}$ beam is captured in a. phase space area of $0.05 \mathrm{ev} \cdot \mathrm{sec} / \mathrm{amu}$. The effects due to the space charge are seen in Figure 7. 200 bins, 20000 representative particles are used in the simulation. $93.8 \%$ of the beam is finally captured. The beam populated phase space area is $0.06 e v \cdot s e c / a m u$.

\section{B. R.F. Capture of the Proton}

The d.c. proton beam of $100 \mu s$ pulse length is assumed being injected into the Booster. Harmonic number of $h=3$ and intensity of $5 \cdot 10^{12}$ per bunch are also assumed. Two different R.F. capture scenarios are explored: the constant $\dot{B}_{0}$ and the programmed $\dot{B}_{0}$.

When $\dot{B}_{0}=1.5 T / \mathrm{sec}$ is assumed during the capturing process, $\hat{V}=90 \mathrm{kV} \phi_{s} \simeq$ $0.05,82 \%$ of the proton beam is captured. The beam populated phase space area is approximately $1.3 \mathrm{ev} \cdot \sec$ (Fig. 9) (Without the space charge, the rate is $86 \%$, Fig. 8). With a constant $\hat{V}$, a very small value of initial $\phi_{s}$ has to be assumed if a high survival rate is desired.

If during the process the $\hat{V}$ and the $\phi_{s}$ are adiabatically increased, say, from $\hat{V}=10 \mathrm{kV}$, $\phi_{s}=0.00$ to $\hat{V}=90 \mathrm{kV}, \phi_{s}=0.05$ in $1 \mathrm{~ms}$ (Fig. $11 \mathrm{a}, b$ ), $96 \%$ of the proton beam can be capture within a much smaller phase space area of $0.9 \mathrm{ev} \cdot \sec$ (Fig. 12,13). The results are summarized in Table 1. The $B_{0}$ and the R.F. frequency are shown in Fig. $14 a, b$.

\section{DISCUSSIONS}

During the R.F. capture on the Booster the effects given by the space charge are by no means negligible. The de-focusing space charge force inevitably causes non-uniformity and diffusion in the longitudinal phase space. However, if the injection pulse is long enough, the R.F. bucket will be filled up with the particles, hence the space charge effects will be averaged and become weaker. The programming of the R.F. voltage and the synchronous phase is very helpful in achieving a higher capture rate and a smaller beam populated phase space area. 

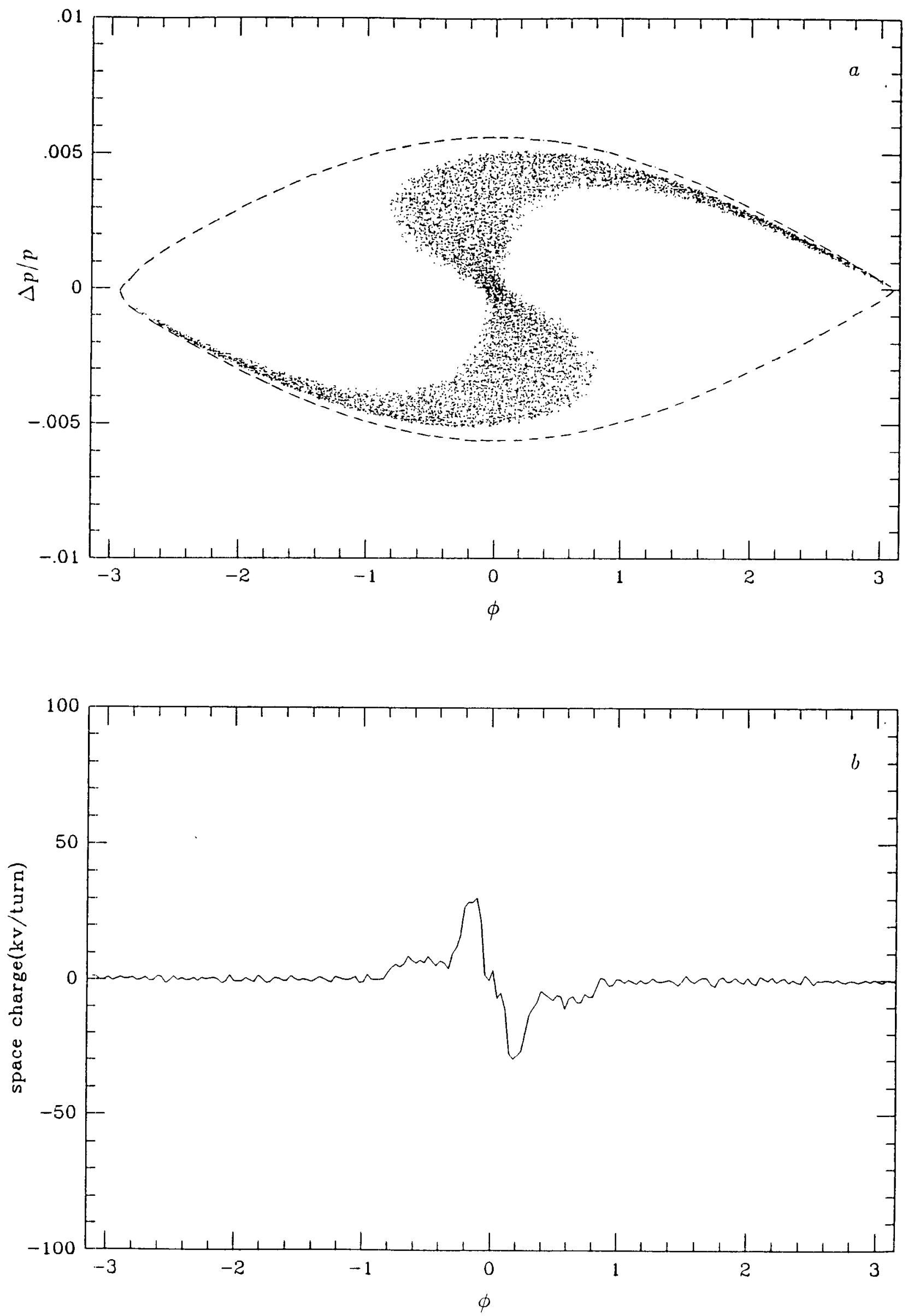

Fig. 1 

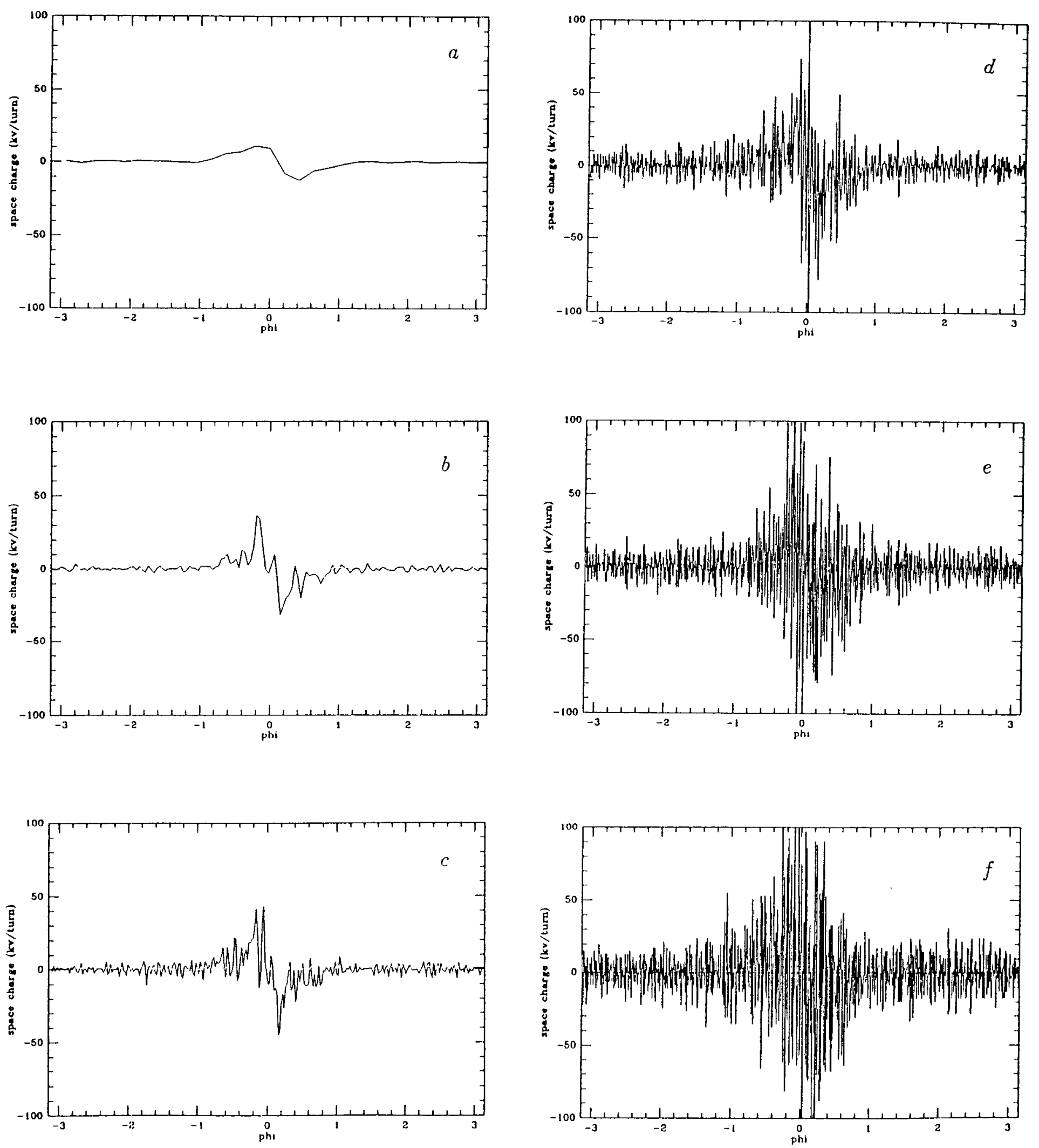

Fig.2 


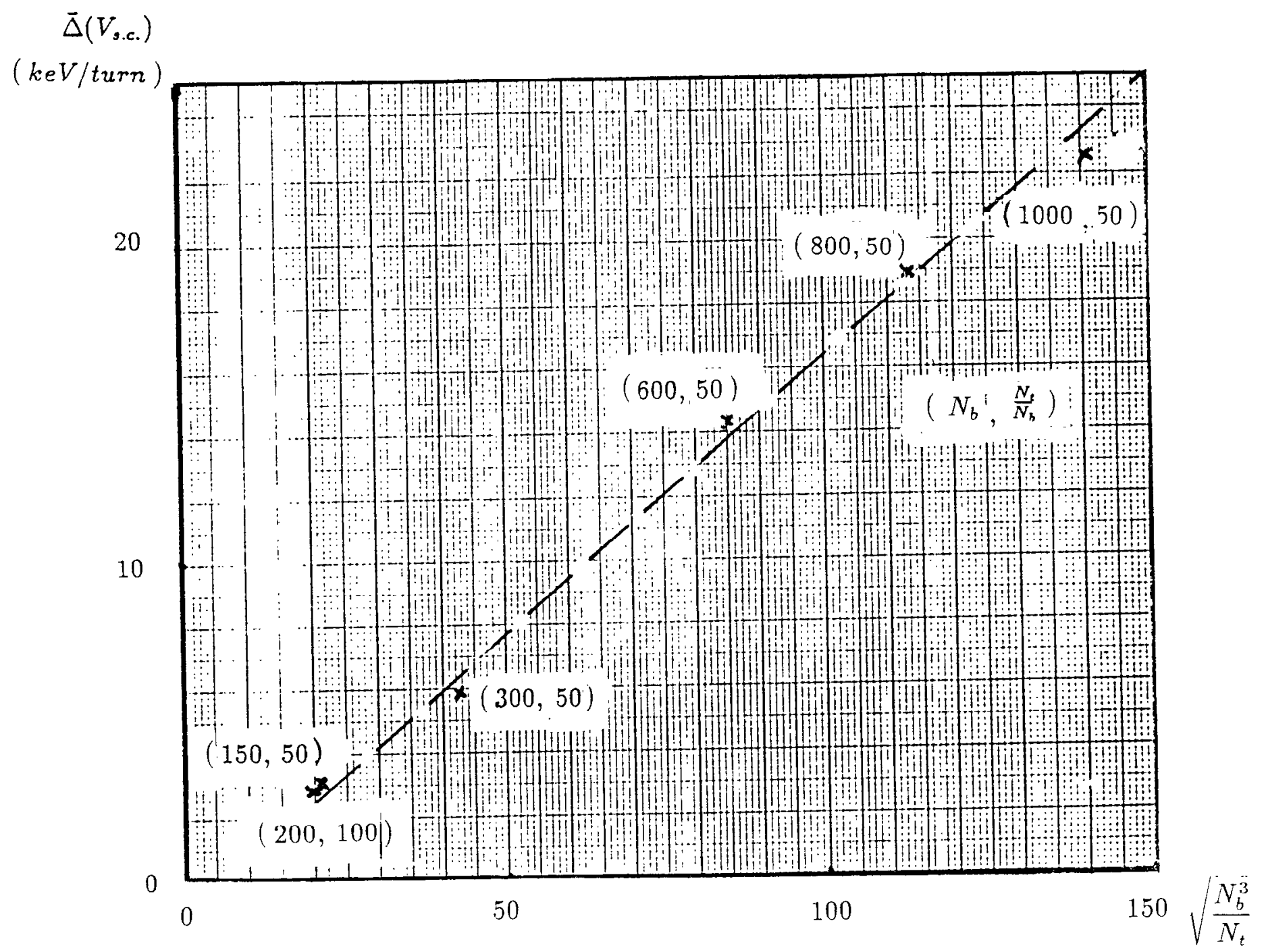

Fig. 3 

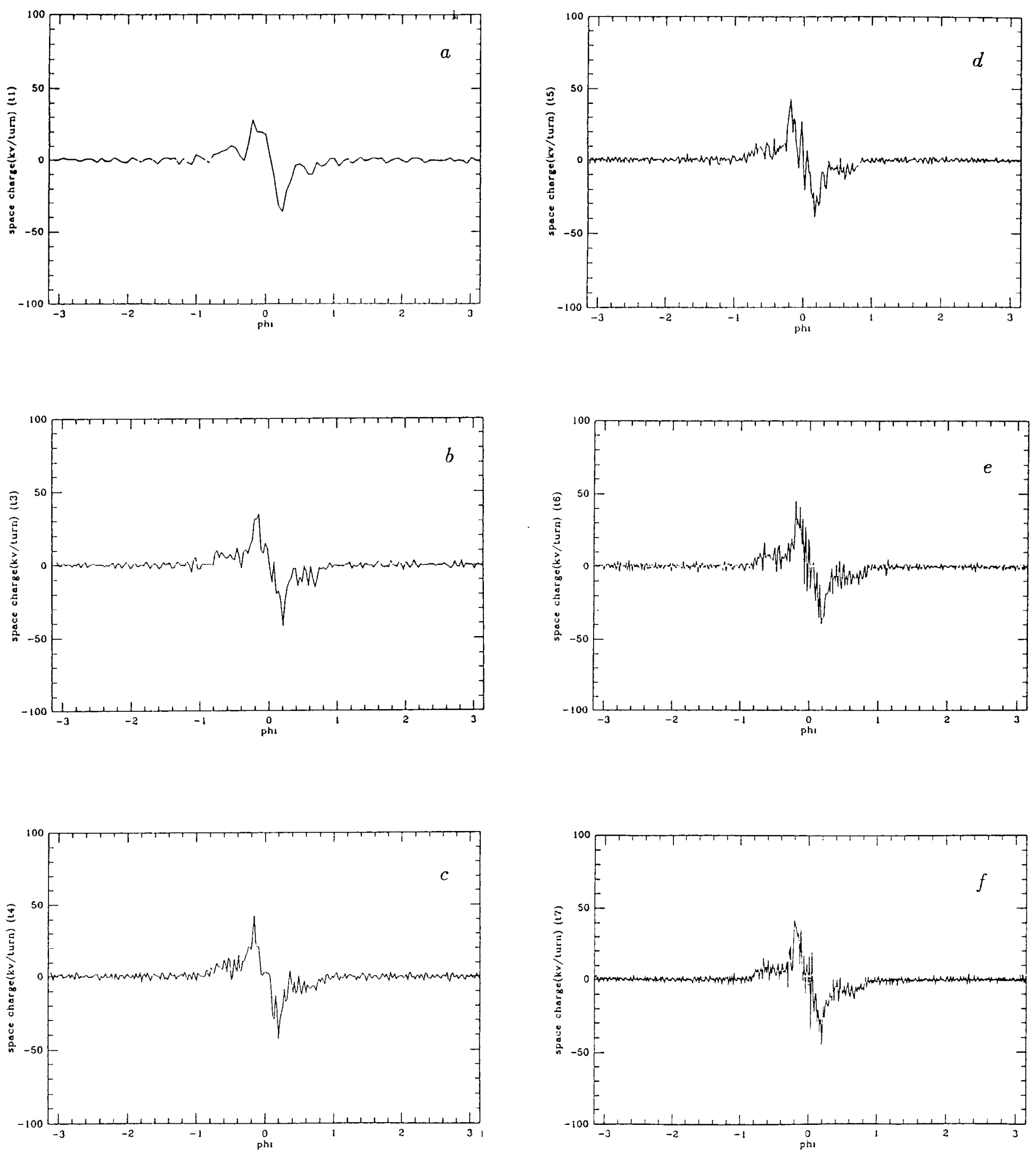

Fig.1 


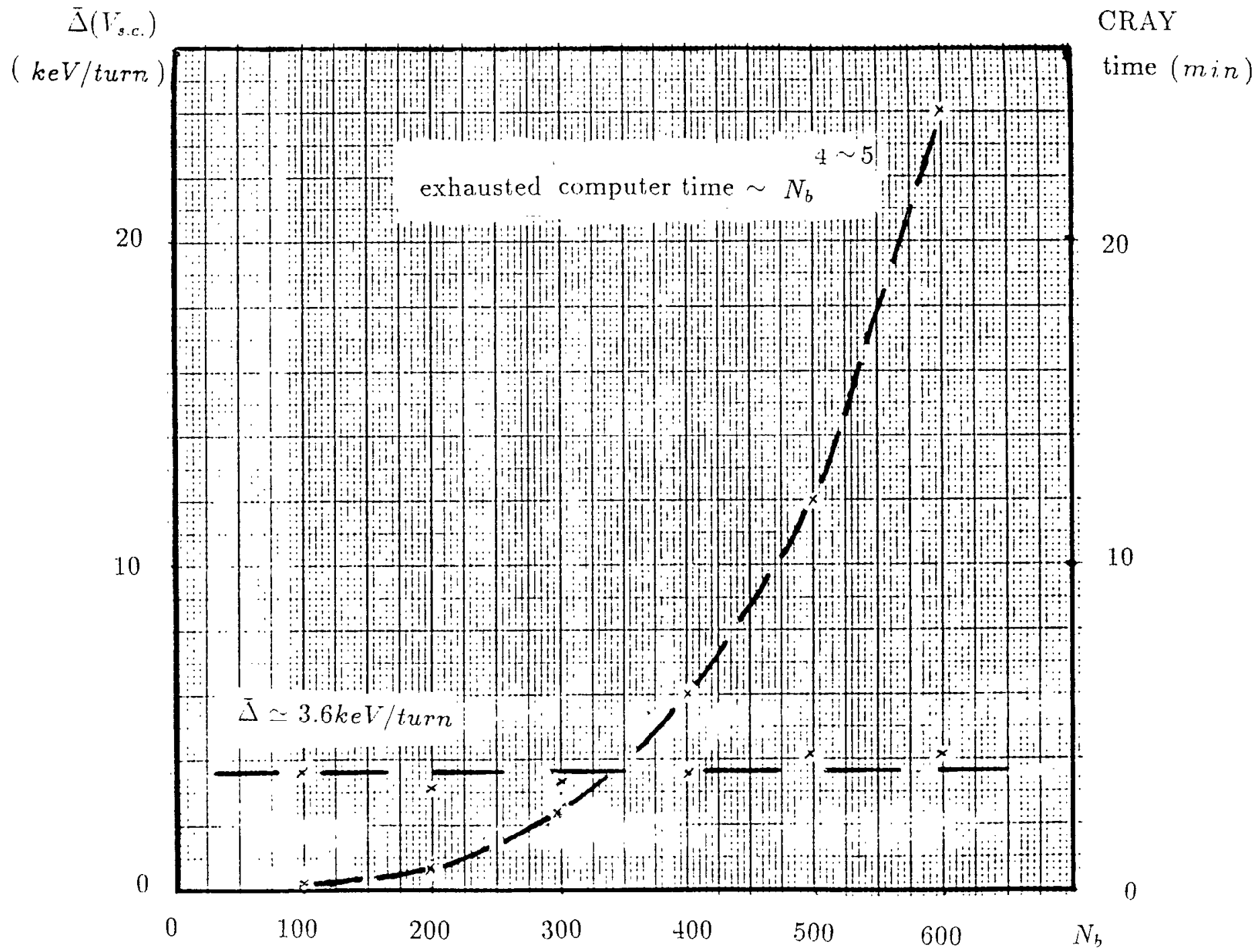

Fig.5 

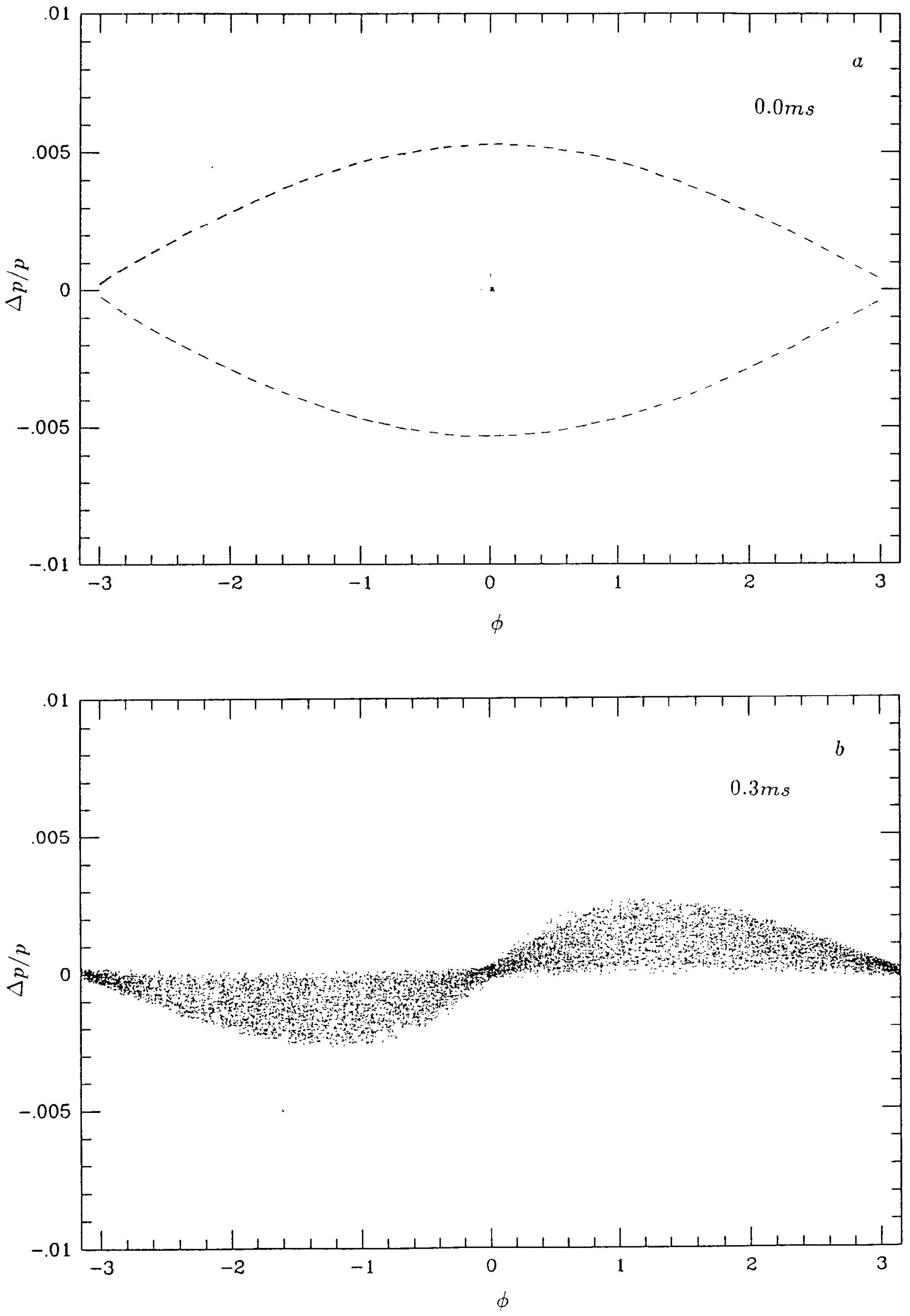

Fig.6 

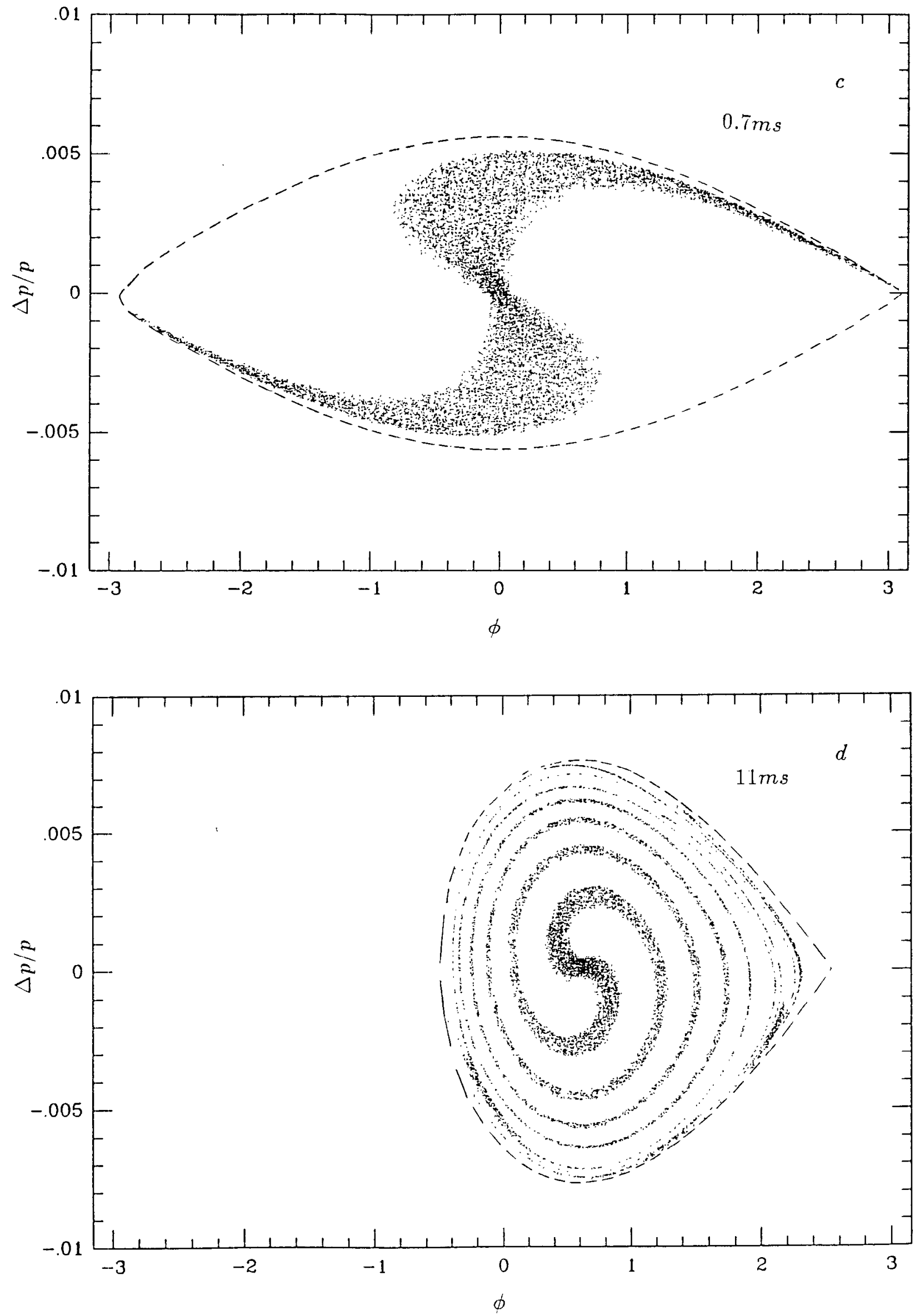

Fig.6 


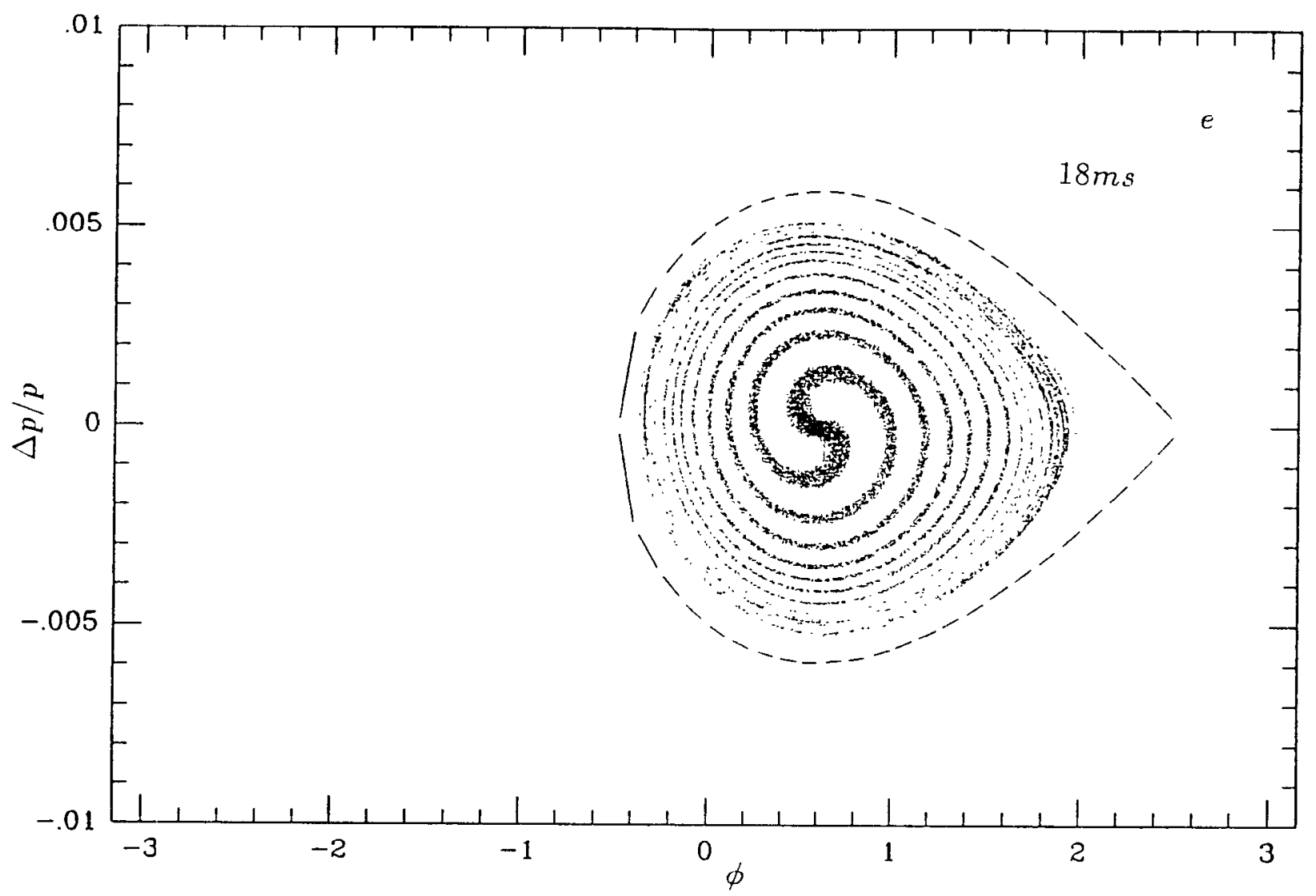

Fig. 6

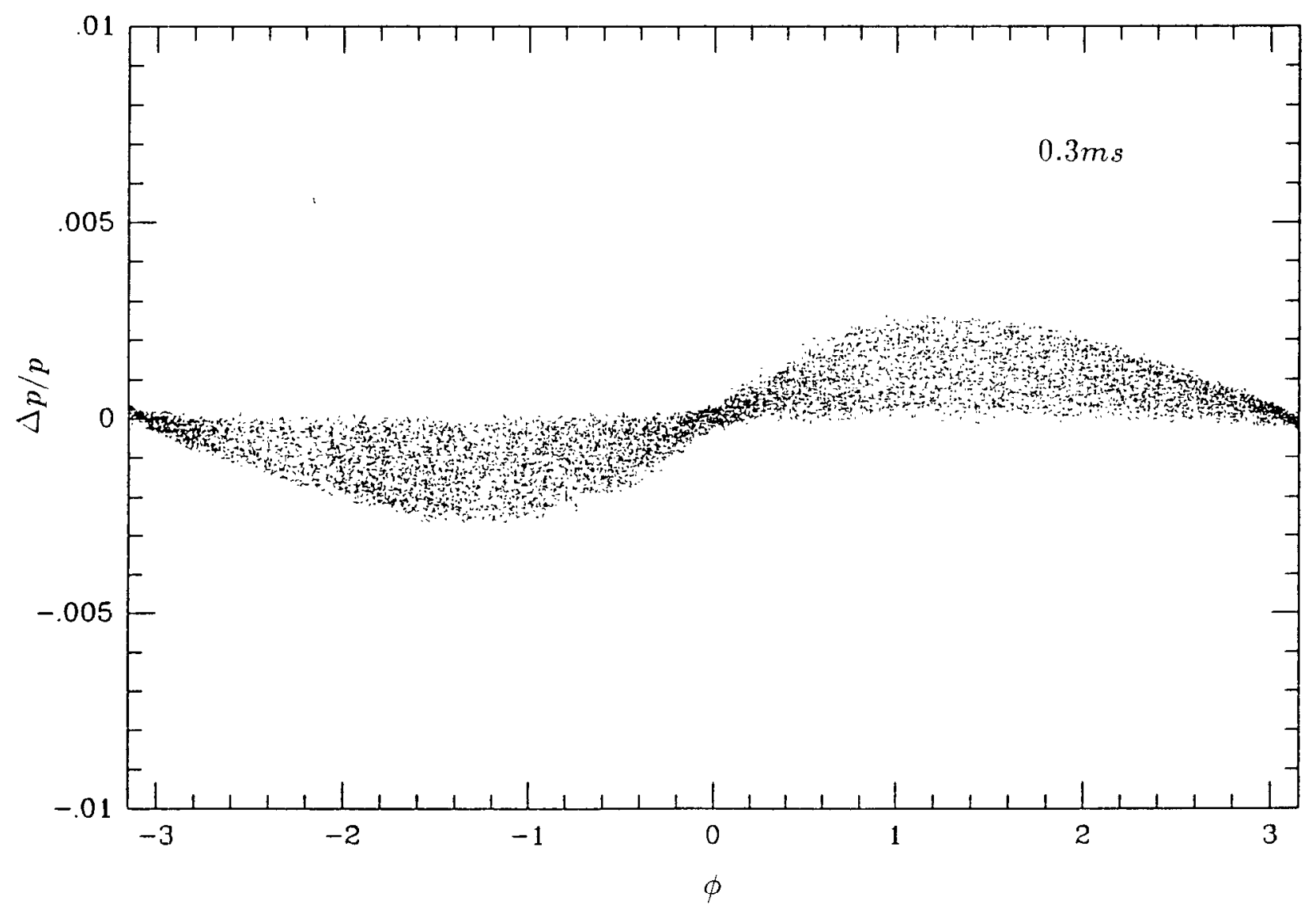

Fig.7a 

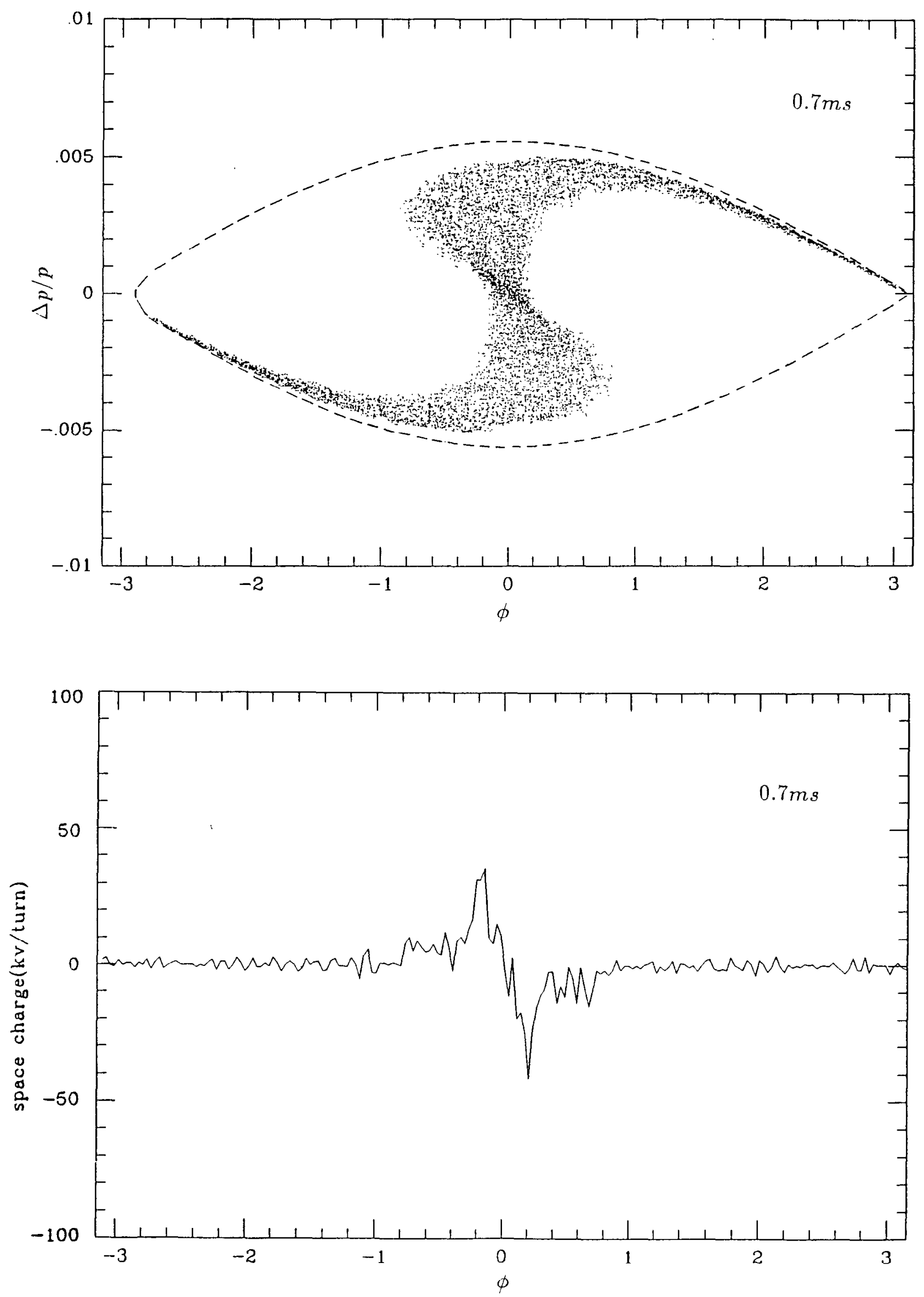

Fig.T 

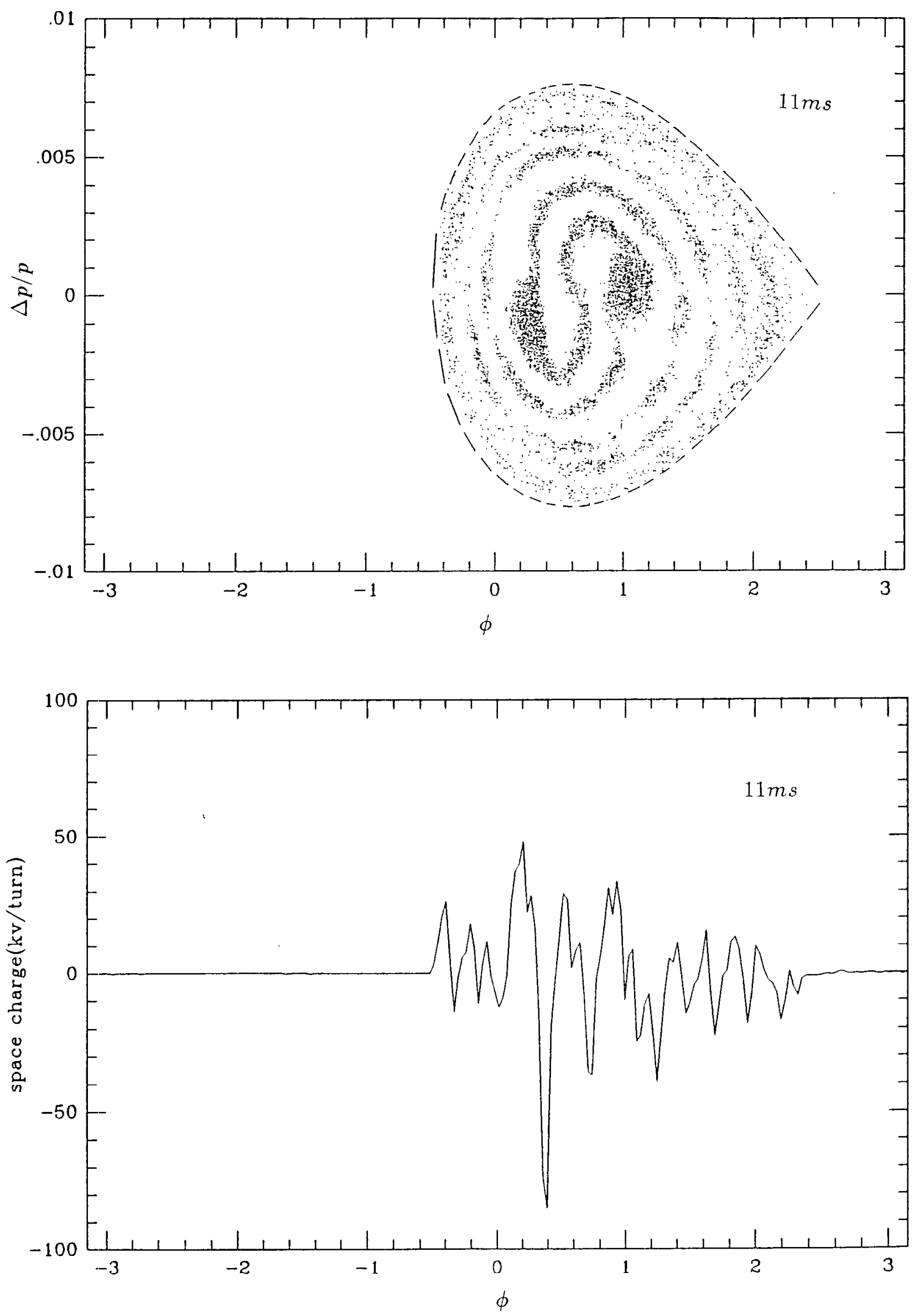

Fig.7c 

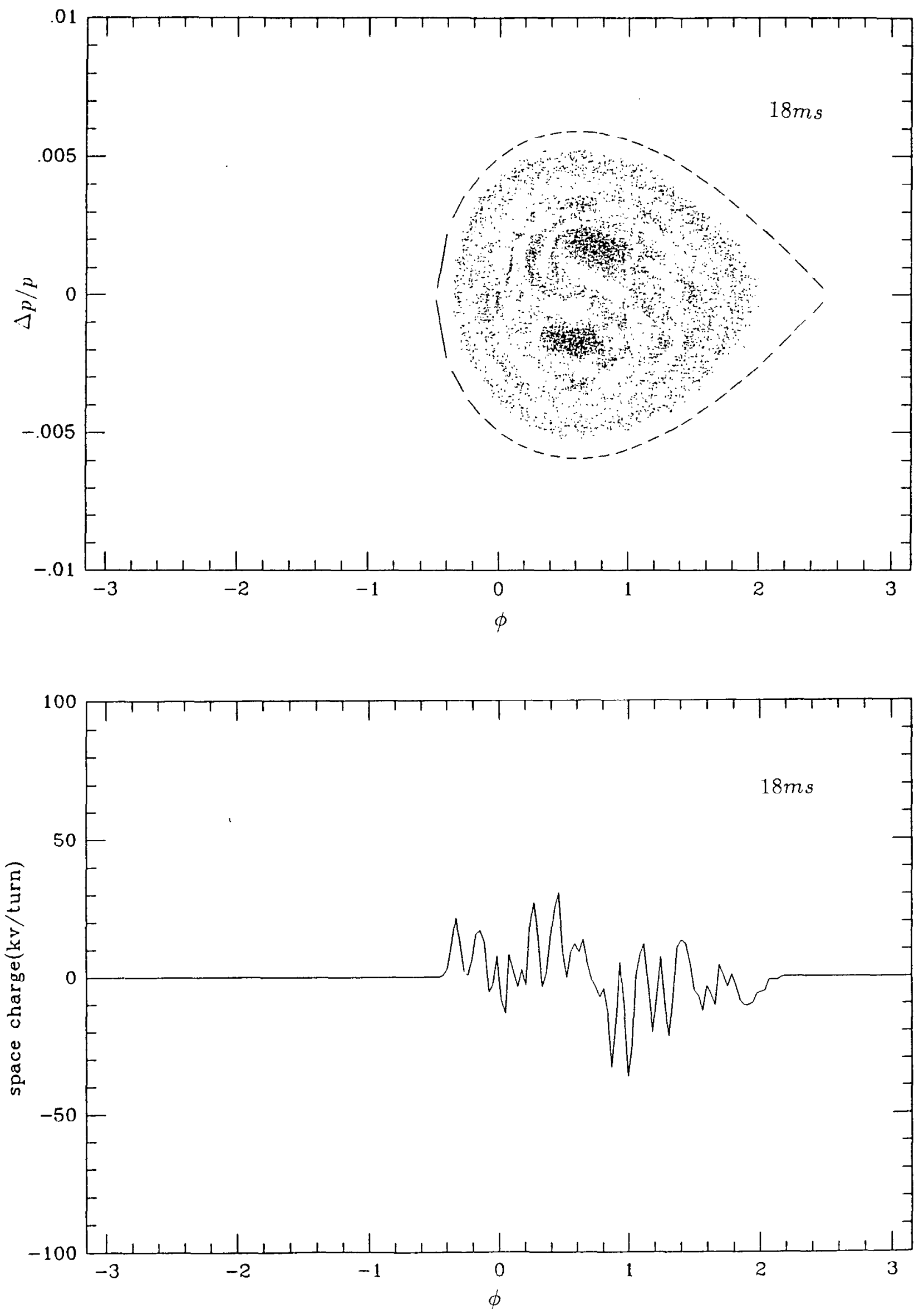

Fig.7d 

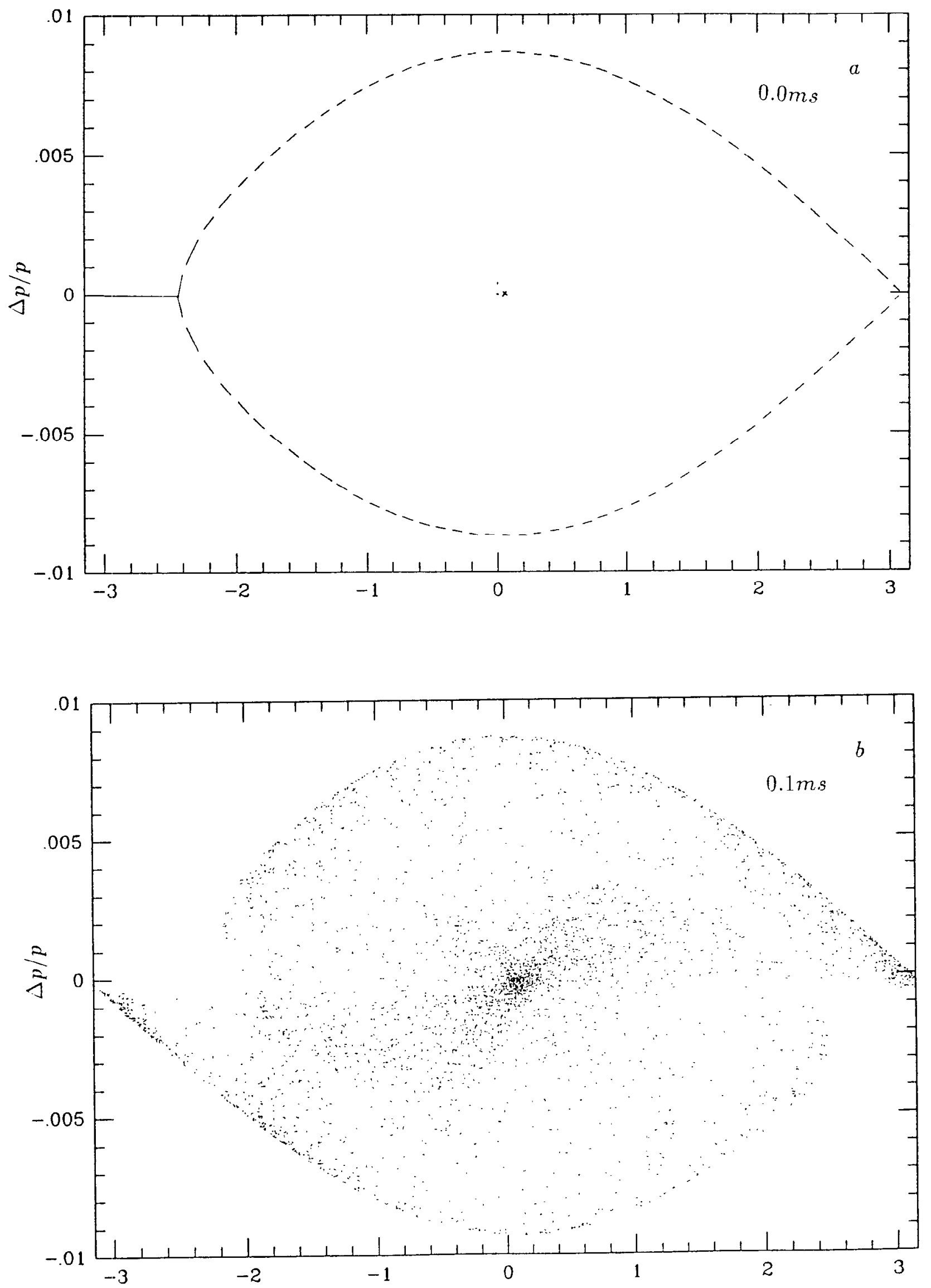

Fig.8 


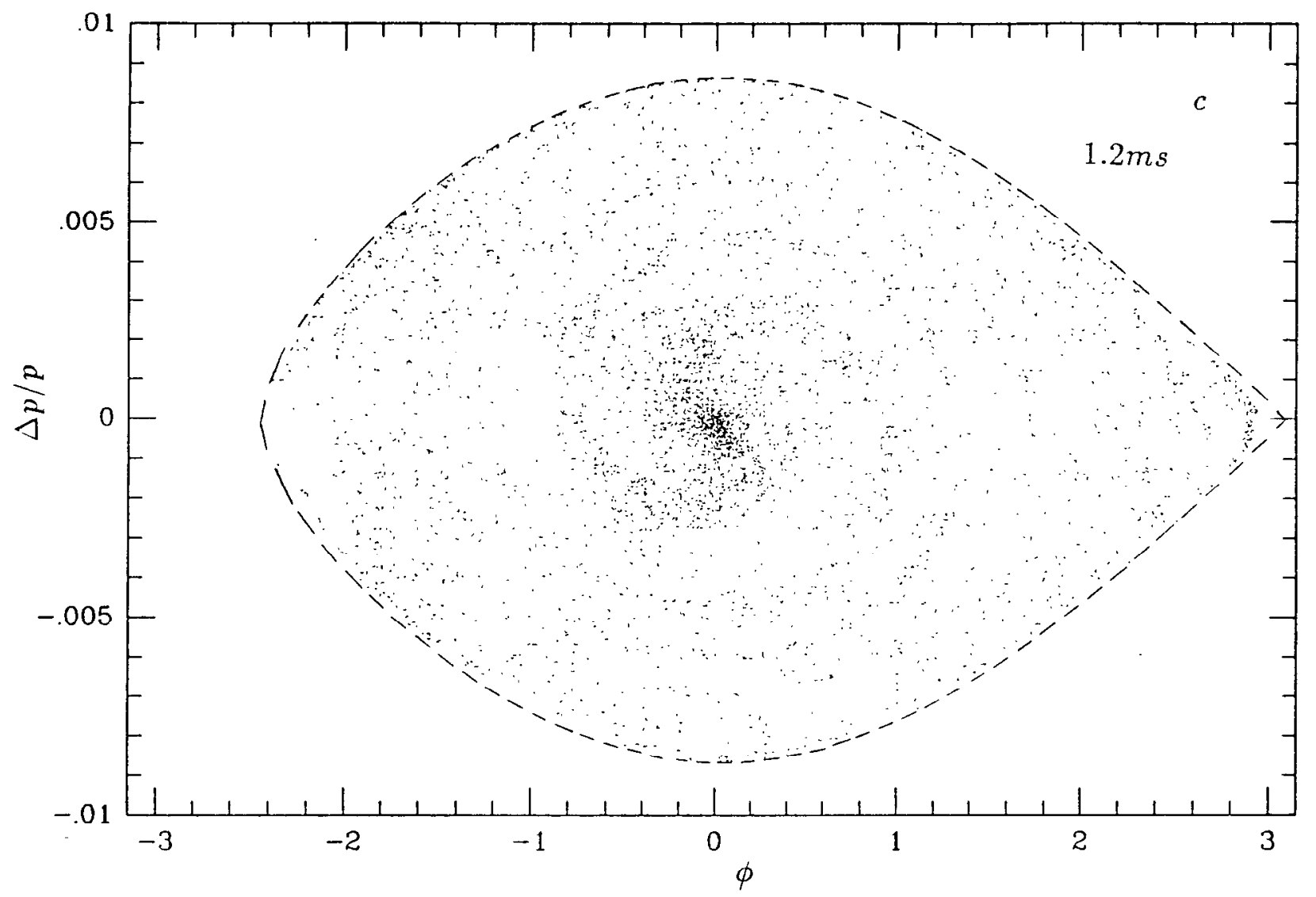

Fig.8

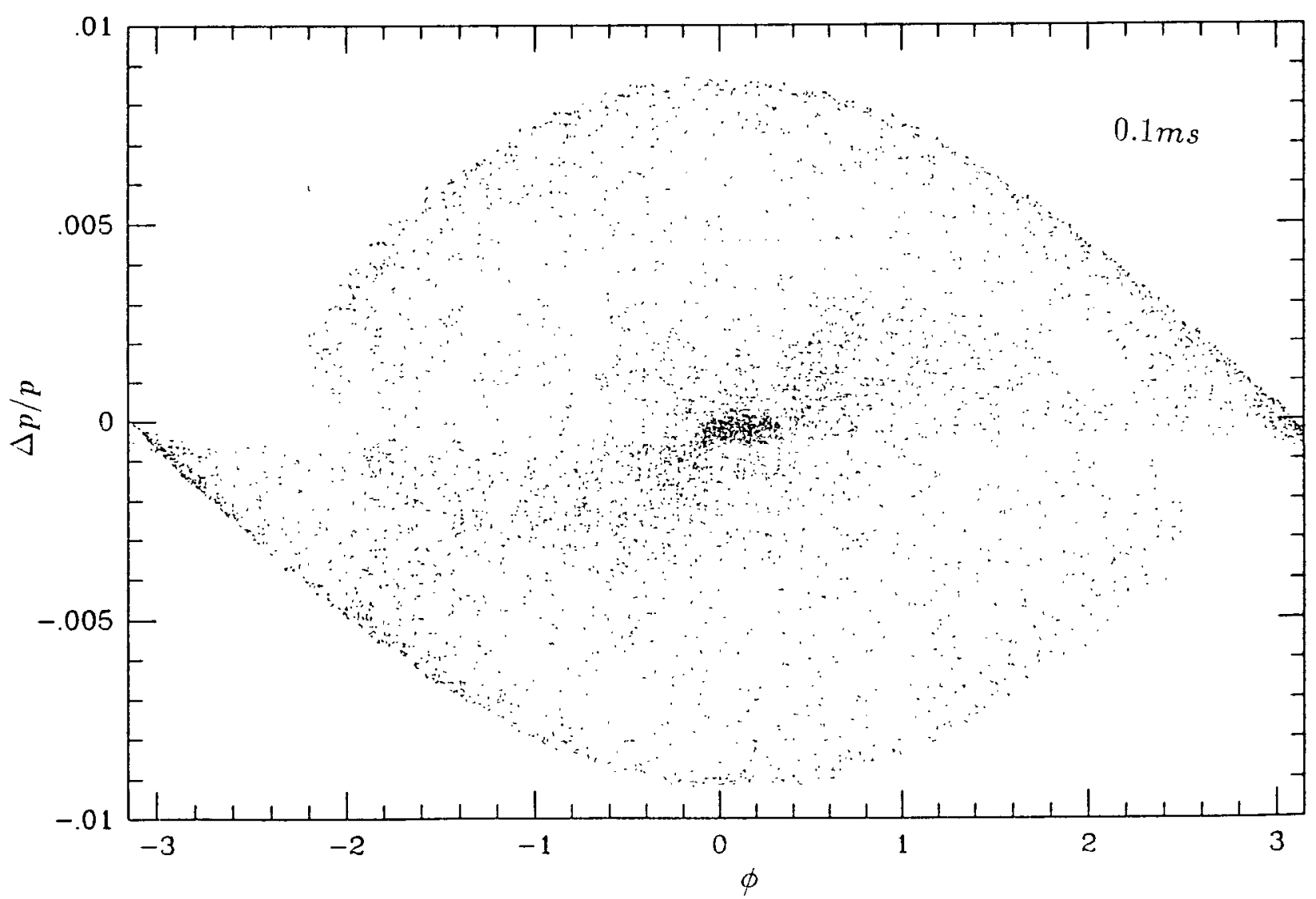

Fig. $9 a$ 

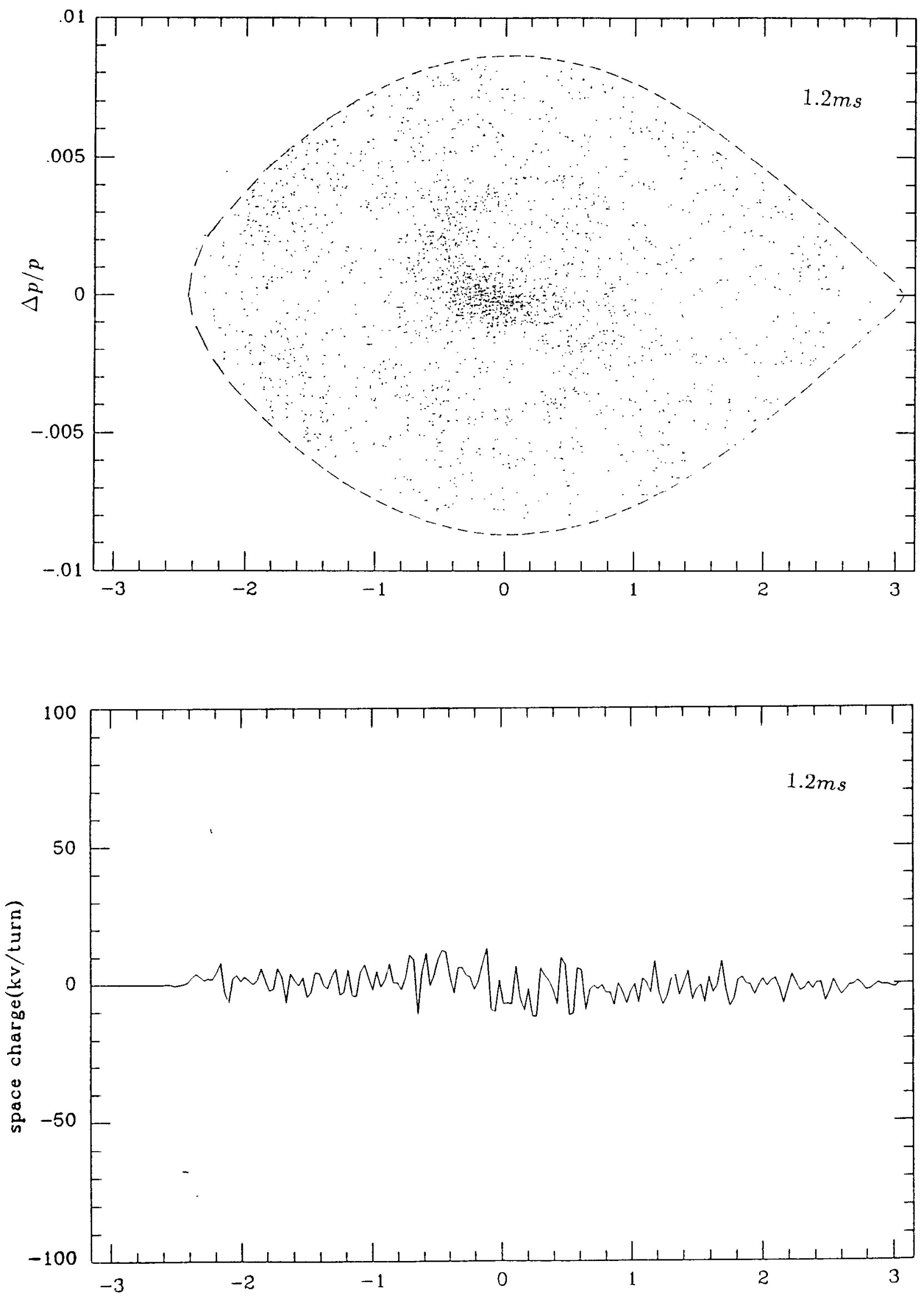

Fig. $9 b$ 


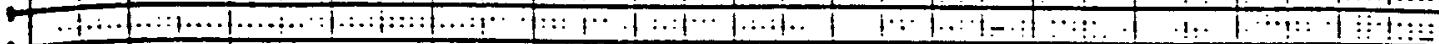
F :

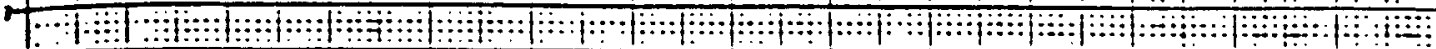
T

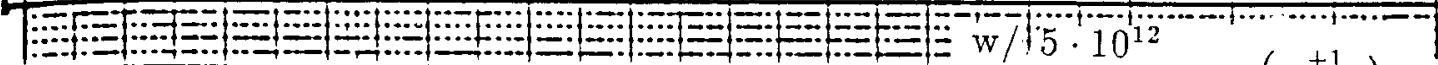

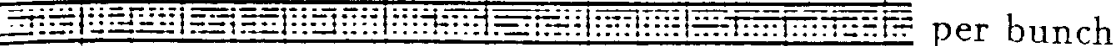
$\left(\mathrm{p}^{+1}\right)$

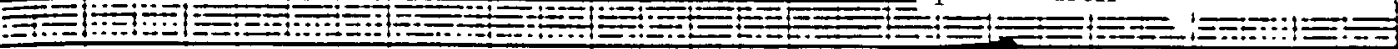

r

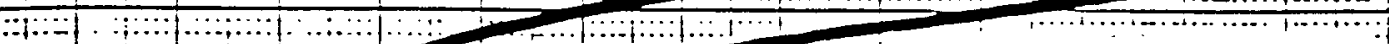
a

10

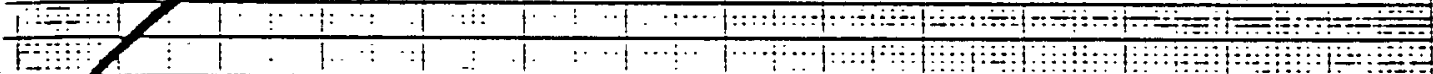

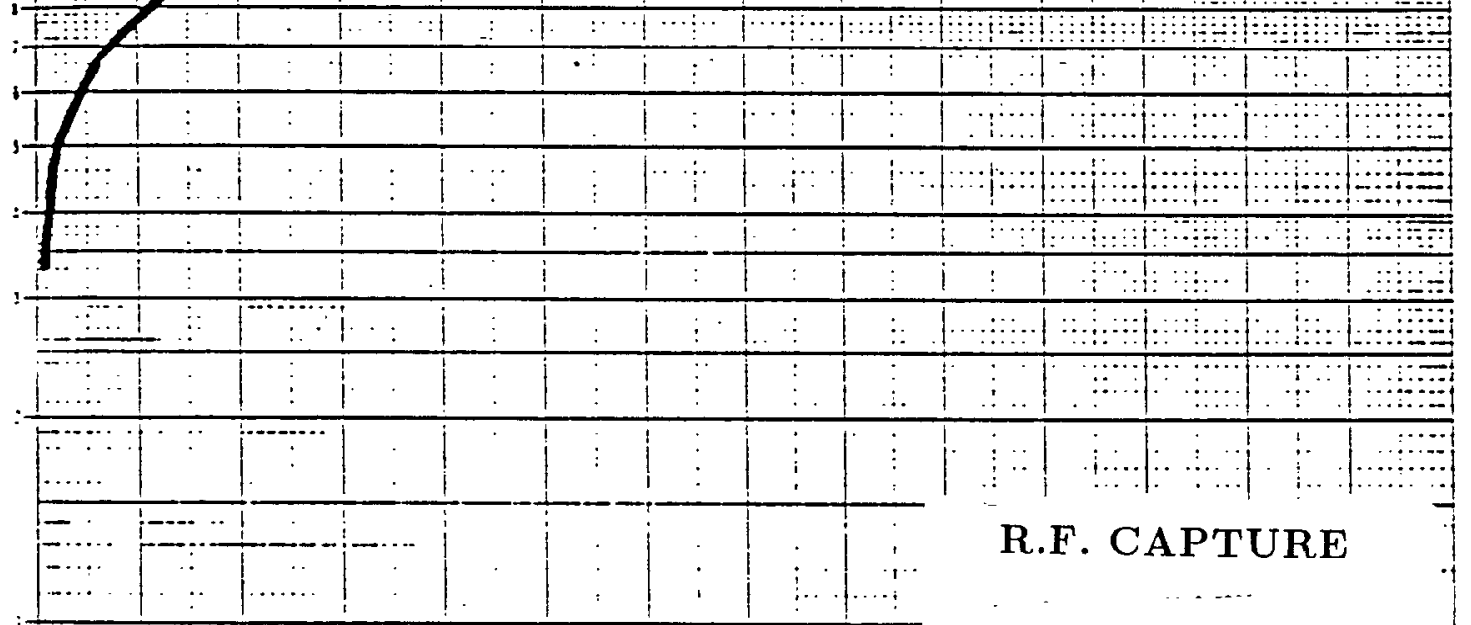

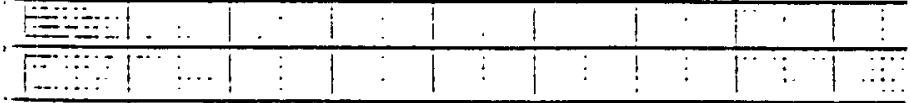

${ }^{197} \mathrm{Au}^{+33} \hat{\mathrm{V}}: 1.6 \mathrm{kV}$

proton $\hat{\mathrm{r}}: 90 \mathrm{kV}$

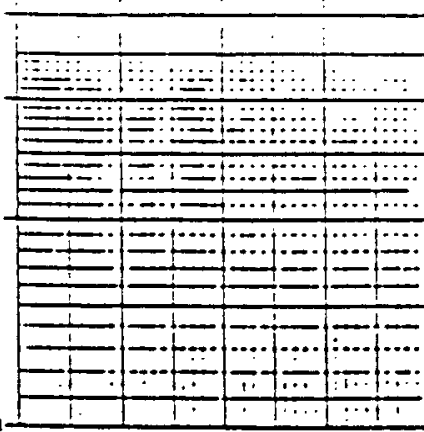

0.0
0.1

synchronous phase $\phi$, 

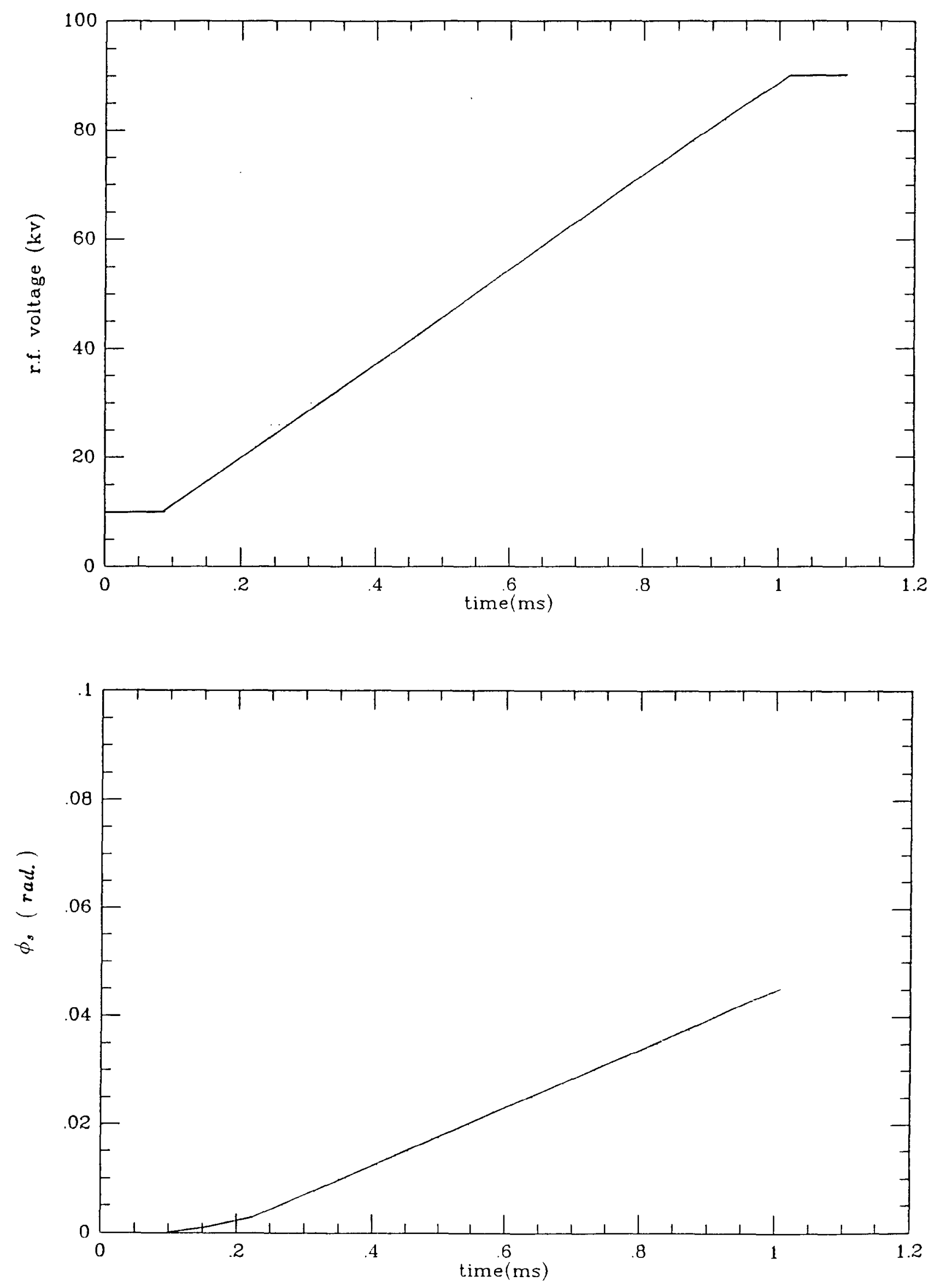

Fig.11 

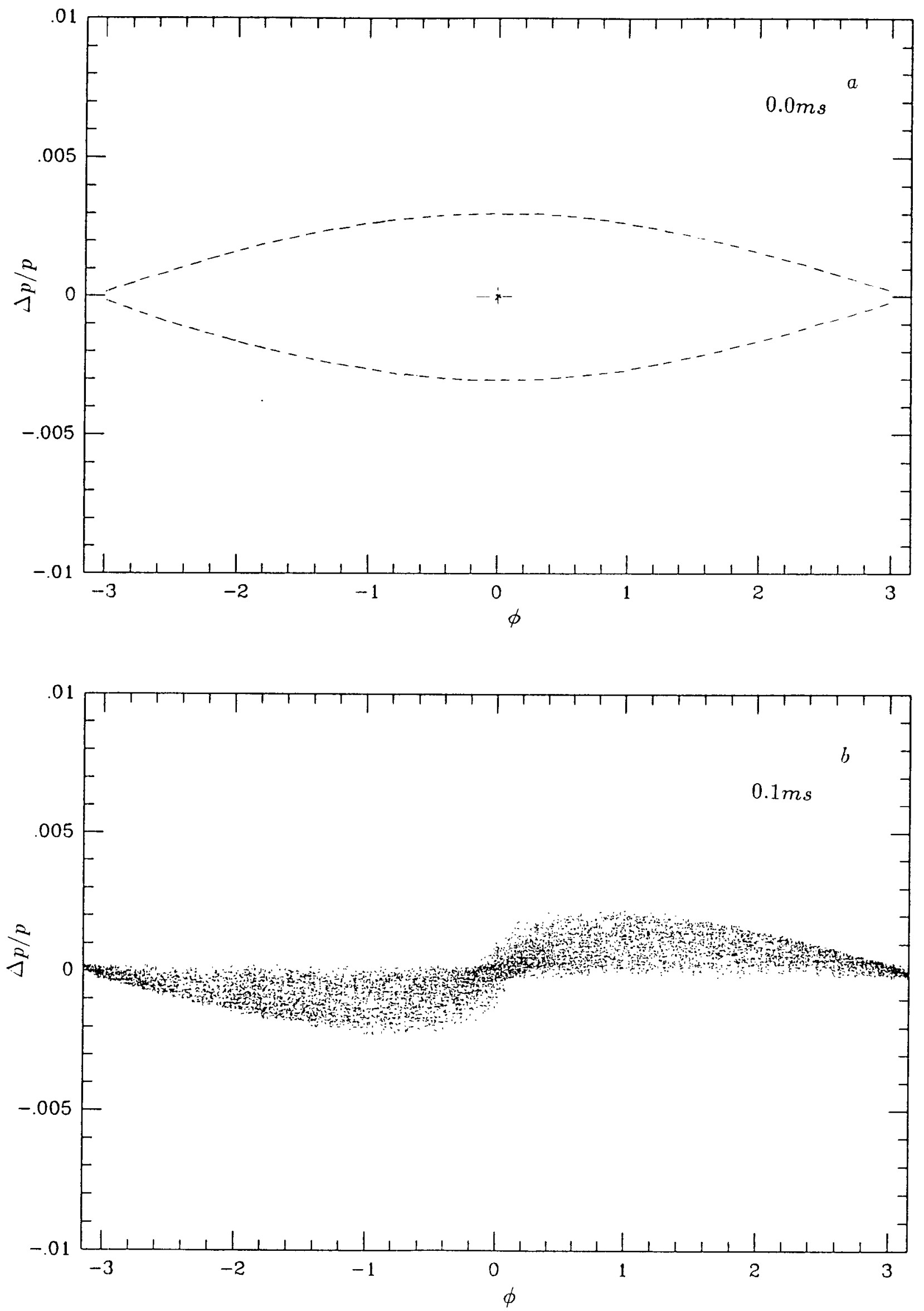

Fig.12 

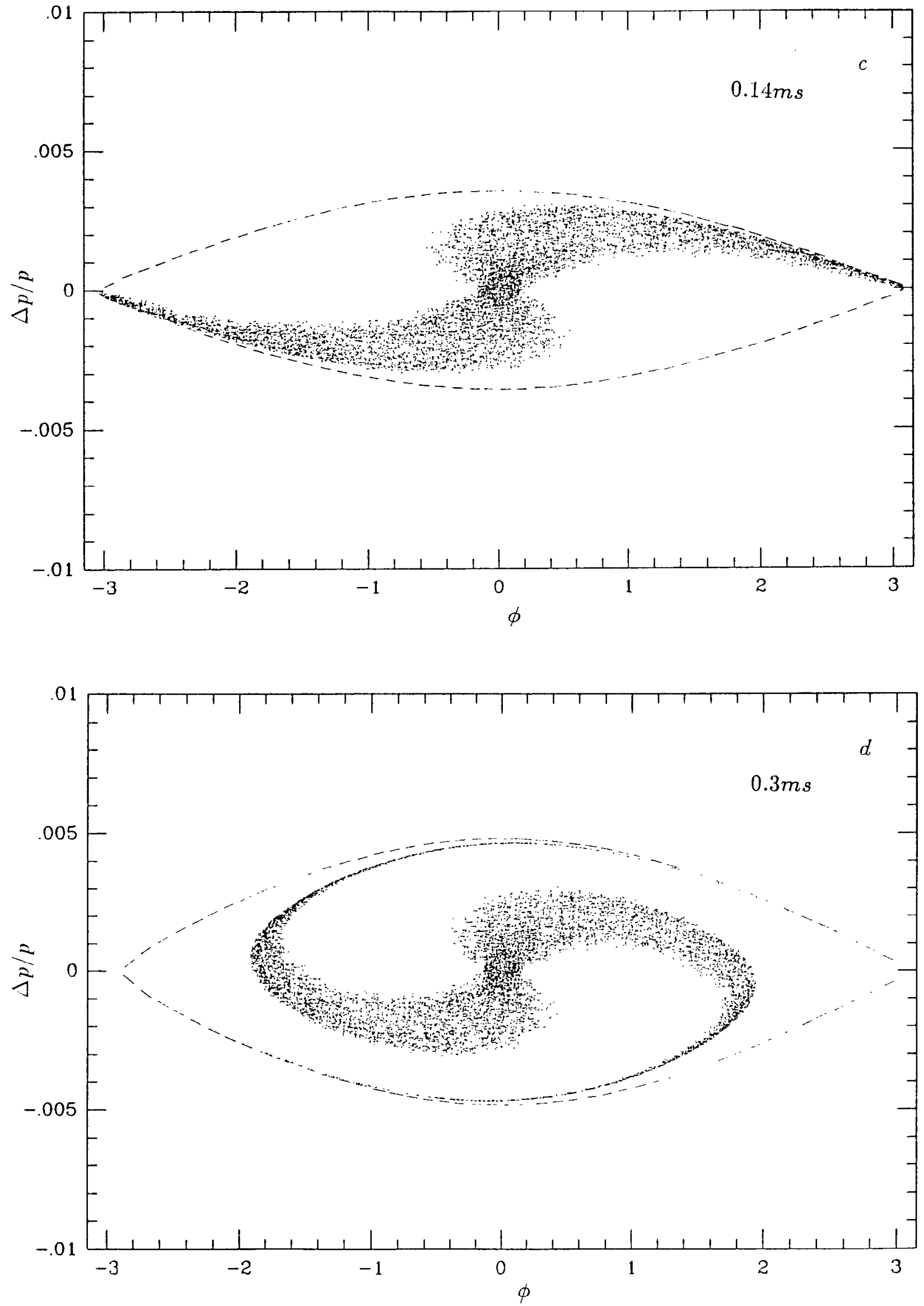

Fig.12 

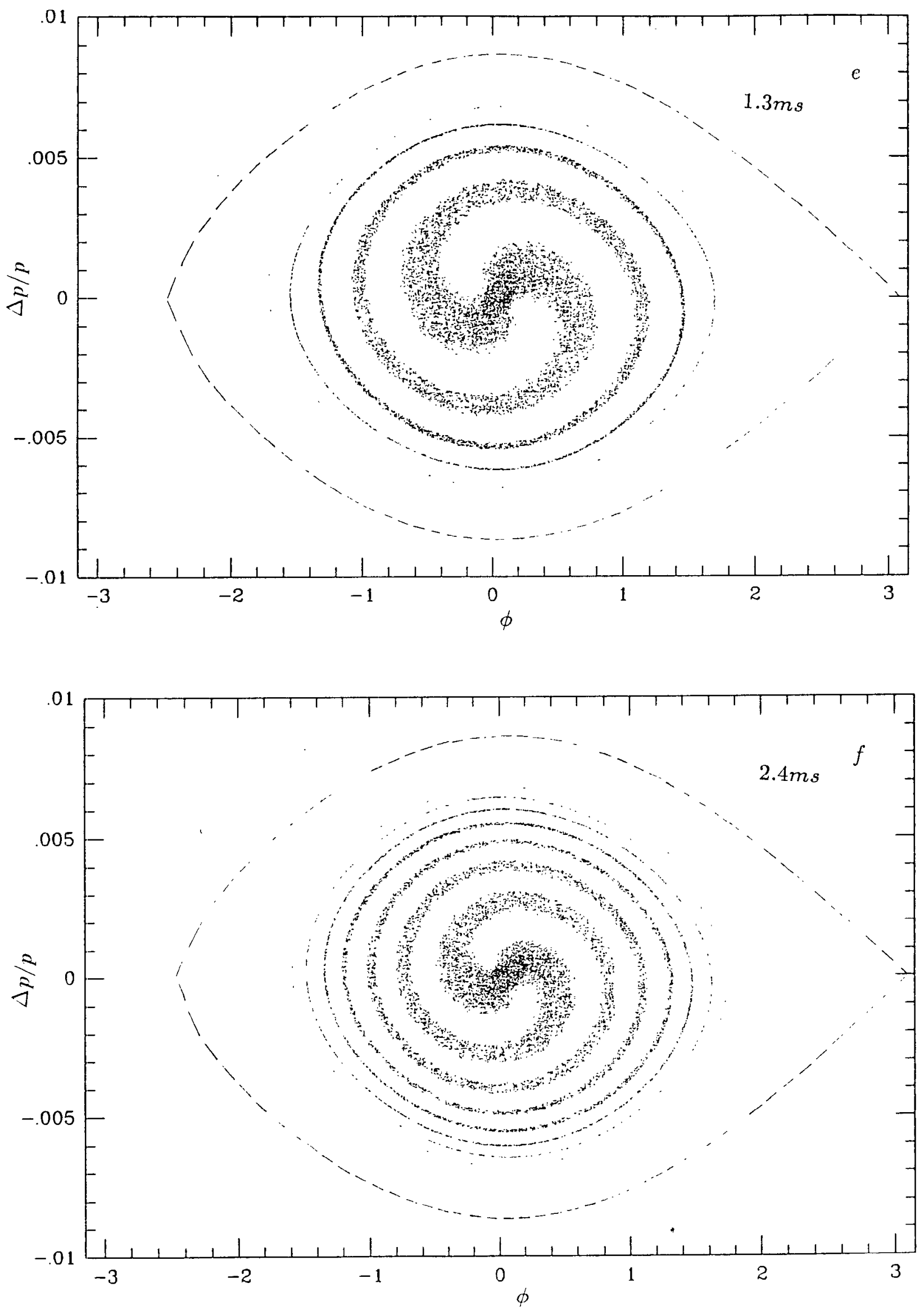

Fig. 12 


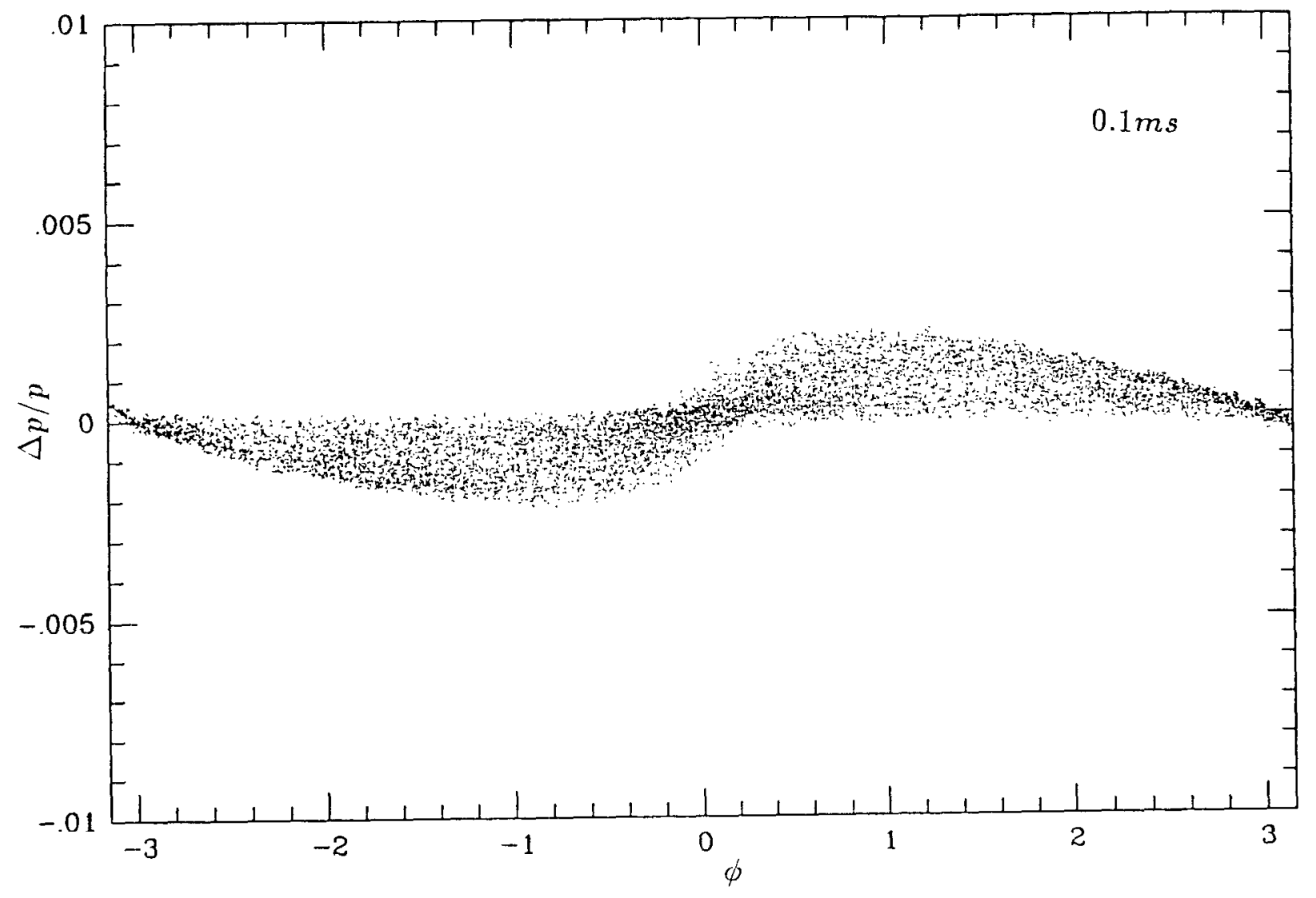

Fig.13a 

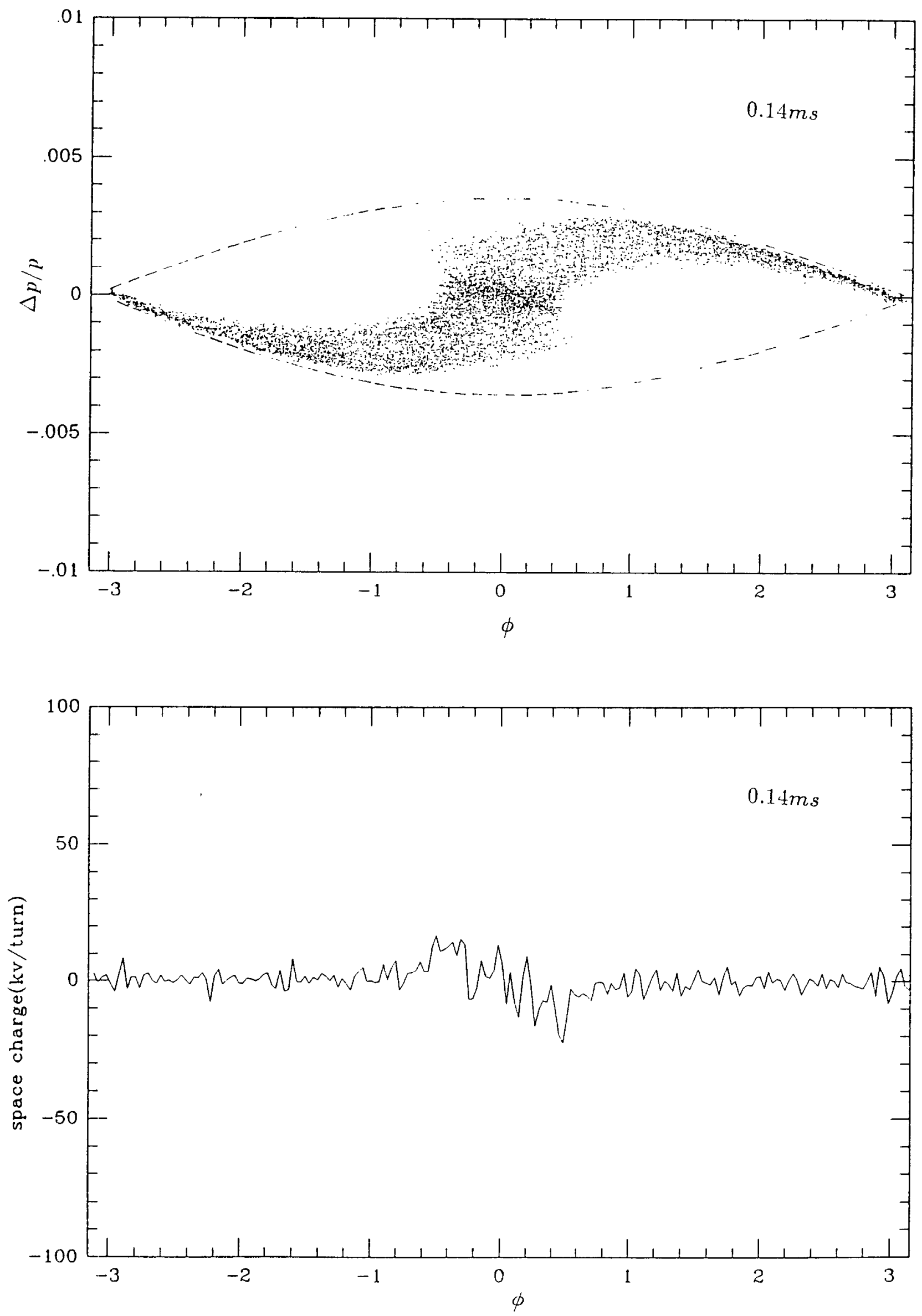

Fig.13b 

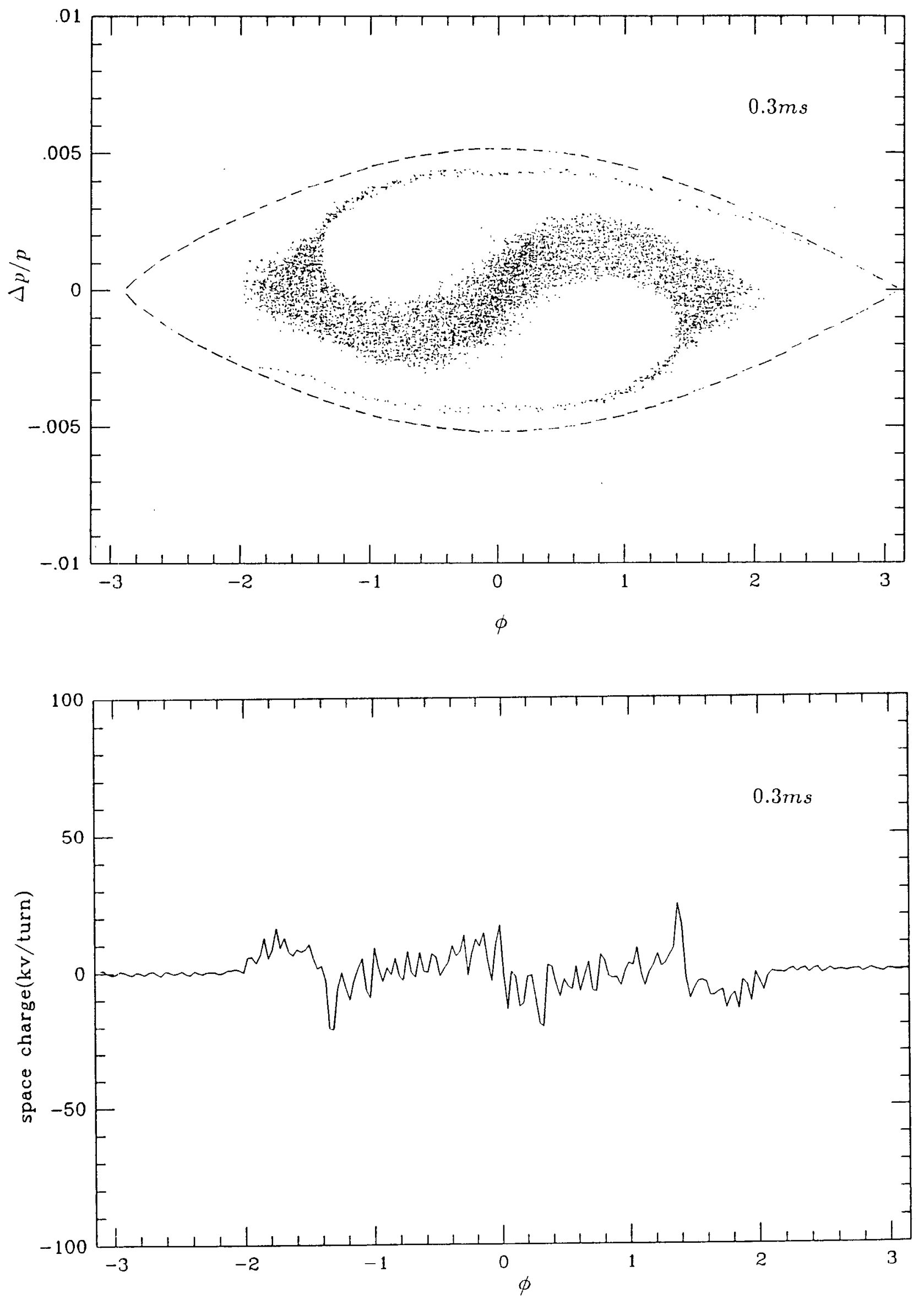

Fig.13c 

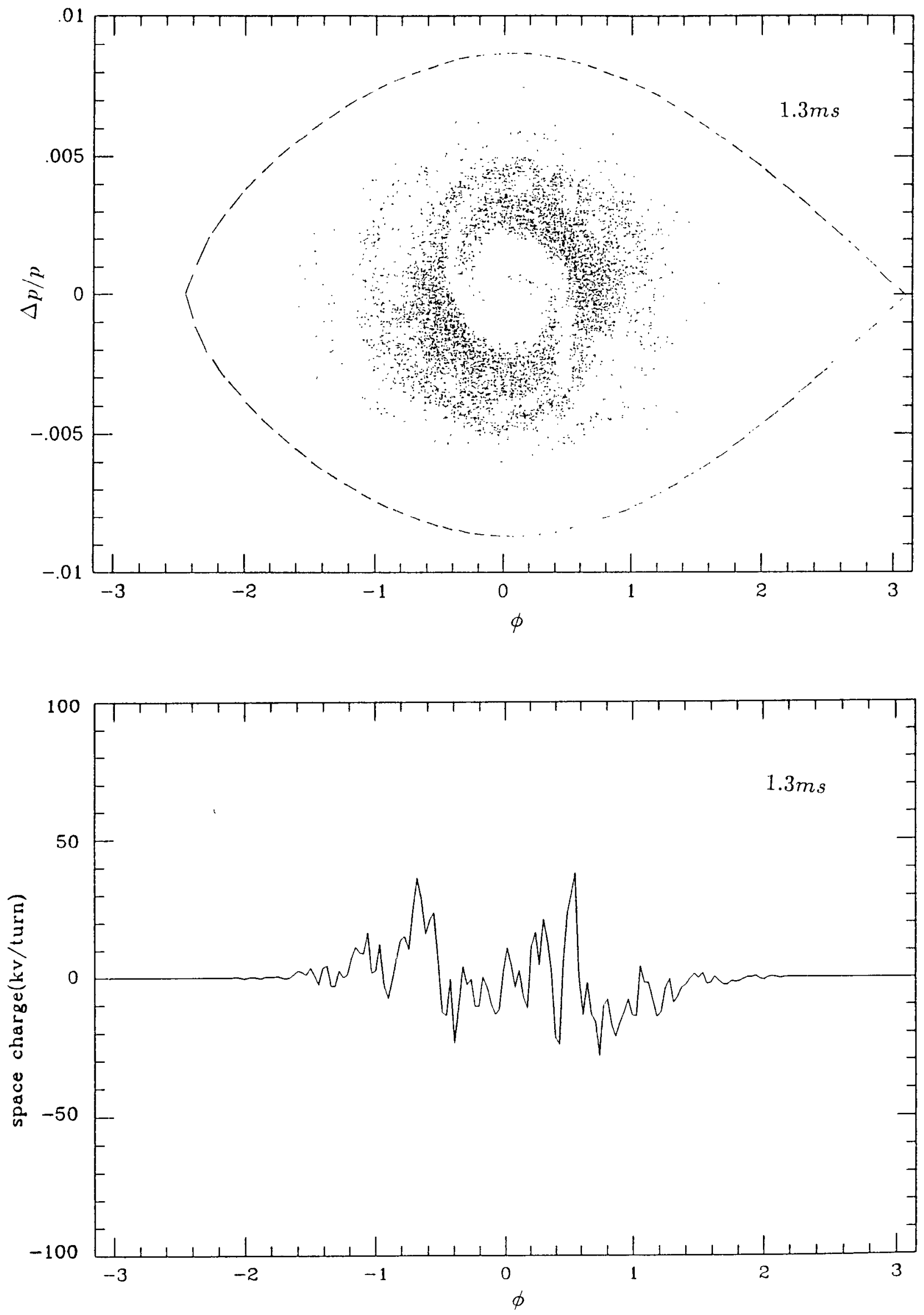

Fig.13d 

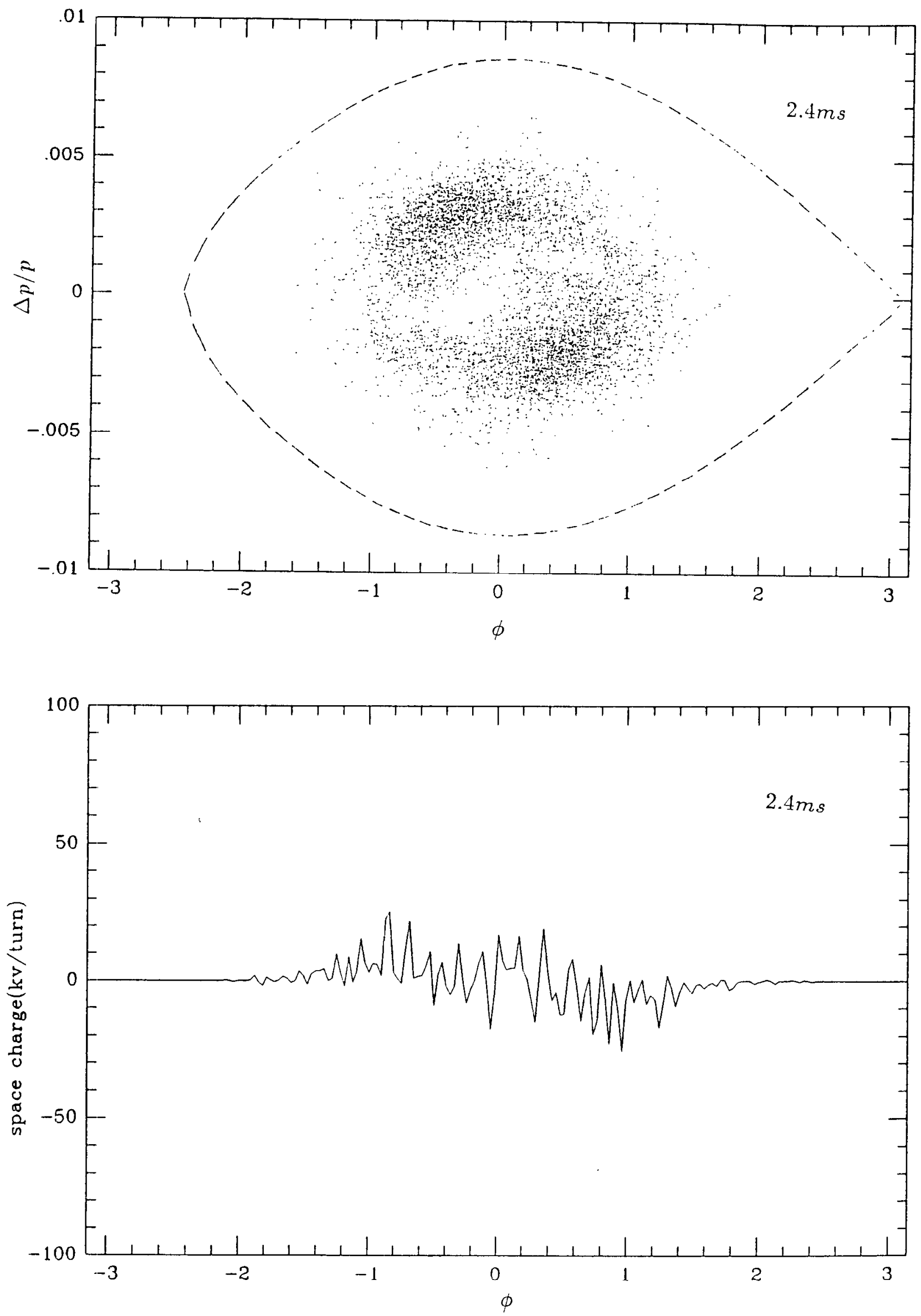

Fig.13e 

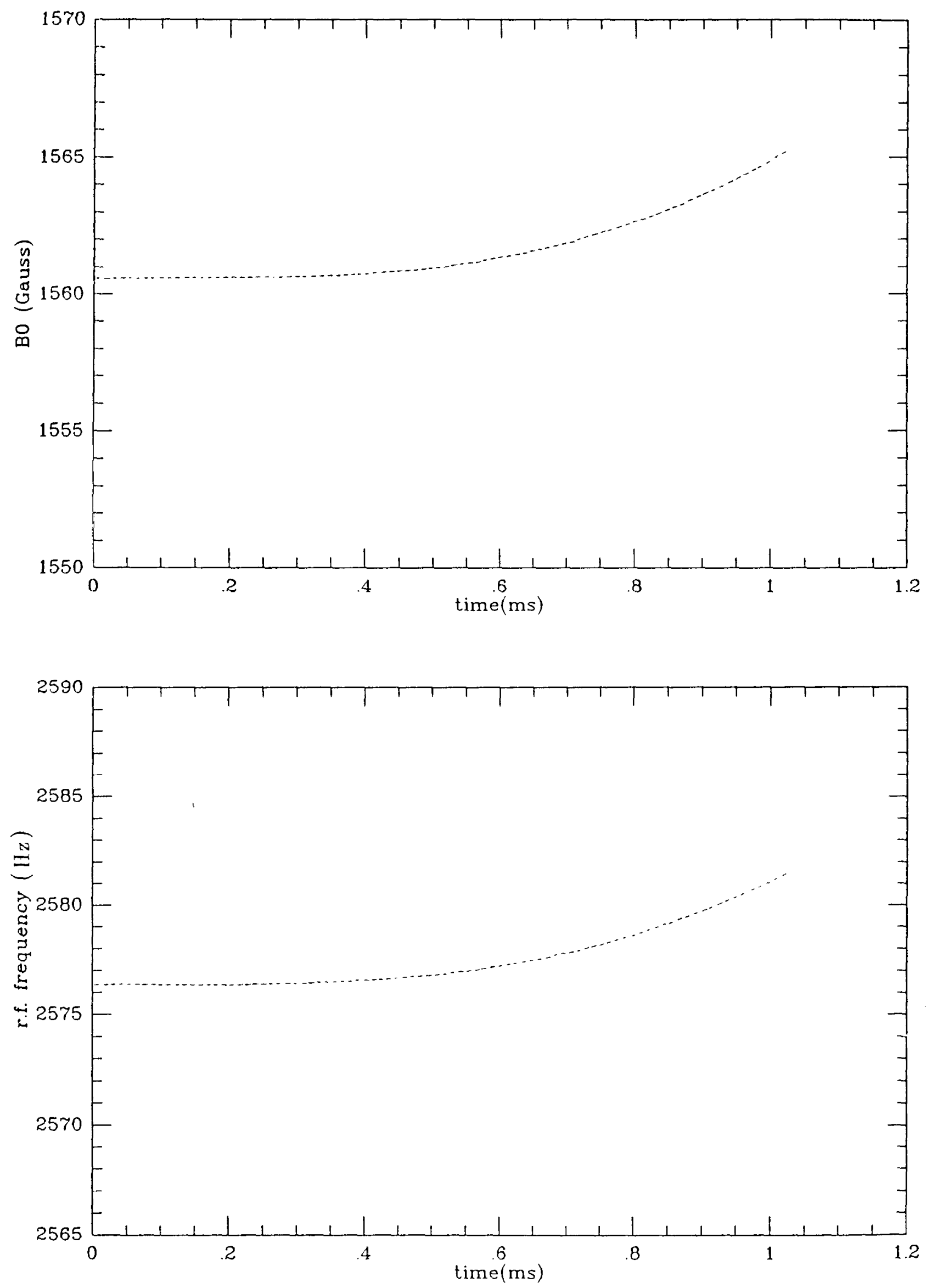

Fig. 14 